\title{
Structure of Aedes aegypti procarboxypeptidase B1 and its binding with Dengue virus for controlling infection
}

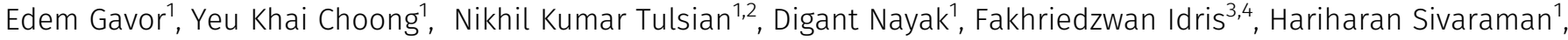 \\ Donald Heng Rong Ting ${ }^{3,4}$, Alonso Sylvie ${ }^{3,4}$, Yu Keung Mok ${ }^{1}$, R Manjunatha Kini ${ }^{1,5}$, J Sivaraman ${ }^{1}$
}

\begin{abstract}
Metallocarboxypeptidases play critical roles in the development of mosquitoes and influence pathogen/parasite infection of the mosquito midgut. Here, we report the crystal structure of Aedes aegypti procarboxypeptidase B1 (PCPBAe1), characterized its substrate specificity and mechanism of binding to and inhibiting Dengue virus (DENV). We show that the activated PCPBAe1 (CPBAe1) hydrolyzes both Arg- and Lys-substrates, which is modulated by residues Asp 251 and $\mathrm{Se}^{239}$. Notably, these residues are conserved in CPBs across mosquito species, possibly required for efficient digestion of basic dietary residues that are necessary for mosquito reproduction and development. Importantly, we characterized the interaction between PCPBAe1 and DENV envelope (E) protein, virus-like particles, and infectious virions. We identified residues $\mathrm{Asp}^{18 \mathrm{~A}}, \mathrm{Glu}^{19 \mathrm{~A}}, \mathrm{Glu}^{85}, \mathrm{Arg}^{87}$, and $\mathrm{Arg}^{89}$ of PCPBAe1 are essential for interaction with DENV. PCPBAe1 maps to the dimeric interface of the E protein domains I/II $\left(\mathrm{Lys}^{64}-\mathrm{Glu}^{84}, \mathrm{Val}^{238}{ }^{23 a l^{252}}\right.$, and $\mathrm{Leu}^{278}{ }^{2 \mathrm{Le}}{ }^{287}$ ). Overall, our studies provide general insights into how the substrate-binding property of mosquito carboxypeptidases could be targeted to potentially control mosquito populations or proposes a mechanism by which PCPBAe1 binds to and inhibits DENV.
\end{abstract}

DOI 10.26508/Isa.202101211 | Received 24 August 2021 | Revised 18 October 2021 | Accepted 19 October 2021 | Published online 8 November 2021

\section{Introduction}

Mosquito-borne viruses such as dengue virus, Zika virus, and chikungunya virus, as well as the malaria parasite constitute a major threat to public health. The mosquito midgut serves as the first entry point for pathogen infection and replication $(1,2)$. The successful establishment of infection in the midgut cells is a prerequisite for pathogen survival and transmission. Studies have shown that some specific proteins in the midgut promote pathogenicity $(3,4,5)$, whereas certain other proteins elicit antiviral/ antiparasitic activities $(6,7)$.
In this regard, the midgut metallocarboxypeptidases (MCPS) have been considered as transmission-blocking vaccine candidates against mosquito and other insect vectors $(4,7,8,9,10,11)$. MCPs are exopeptidases involved in numerous processes, including digestion, blood coagulation/fibrinolysis, inflammation, and pro-hormone and neuropeptide processing (12). In mosquitoes, the A/B subfamily of MCPs (including carboxypeptidases A and $\mathrm{B}$; $\mathrm{CPB}$ and $\mathrm{CPA}$, respectively) is primarily responsible for dietary blood digestion $(3,13,14)$ for the release of amino acid-rich nutrients required for egg development $(4,5,15,16)$. As such, MCPs are important targets for mosquito population control. MCPs also play a significant role in parasite transmission in mosquitoes. For example, the Anopheles midgut CPB1 has been shown to enhance malaria parasite transmission in various Anopheles mosquitoes (4, $5,16)$. Active immunization with recombinant Anopheles gambiae procarboxypeptidase B1 (PCPBAg1) protected mice against Plasmodium berghei challenge (4). In addition, mosquitoes fed with anti-PCPBAg1 antibodies exhibited reduced reproductive capacity (5), a secondary effect of a CPB-based transmission-blocking vaccine that could likely suppress mosquito populations. This effect was also observed using inhibitors against mosquito dipeptidases. Similarly, the infection rate of Plasmodium falciparum gametocytes in mosquitoes was significantly reduced after treatment with anti-CPBAg1 antibodies $(5,16)$. Besides, inhibitors against peptidyl dipeptidase A administered to both An. gambiae and Ae. aegypti mosquito larvae led to stunted growth, reduced fecundity, and mortality (17).

In the midgut of Ae. aegypti mosquito, procarboxypeptidase B1 (CPB1 or PCPBAe1) takes on a different role in pathogen infection, interacting with the DENV-2 envelope (E) protein/virion and impeding viral packaging, maturation, and release from the midgut (6, 7). The interaction between PCPBAe1 and the E protein/virion can also inhibit viral morphogenesis and affect viral glycoprotein processing (7). The overall impact is the release of low titers of mature viruses. Moreover, another procarboxypeptidase $\mathrm{A}$ homolog in Ae. aegypti interacts with the DENV capsid protein (18); albeit, the

\footnotetext{
${ }^{1}$ Department of Biological Sciences, National University of Singapore, Singapore ${ }^{2}$ Department of Biochemistry, Yong Loo Lin School of Medicine, National University of Singapore, Singapore ${ }^{3}$ Infectious Diseases Translational Research Programme, Department of Microbiology and Immunology, Yong Loo Lin School of Medicine, National University of Singapore, Singapore ${ }^{4}$ Immunology Programme, Life Sciences Institute, National University of Singapore, Singapore ${ }^{5}$ Department of Pharmacology, Yong Loo Lin School of Medicine, National University of Singapore, Singapore
}

Correspondence: dbsjayar@nus.edu.sg 
role of this interaction has yet to be established. Furthermore, the Ae. aegypti carboxypeptidase gene promoters drive the expression of genes that hinder parasite development in the guts of transgenic mosquitoes (19).

Despite the crucial roles of MCPs in mosquitoes, the structural and molecular mechanisms associated with these proteases are unclear. Here, we characterized the enzymatic activity of the mature peptidase domain (CPBAe1) and determined the structure of the full-length (FL)-PCPBAe1 (proenzyme) at 2.08- $\AA$ resolution. The results provide insights into the substrate specificity of this enzyme and show how this enzyme differs from other non-mosquito insects carboxypeptidases (11). We characterized the interactions between the recombinant PCPBAe1 and DENV-2 envelope (E)-protein, viruslike particles (VLPS), and infectious virions of different serotypes and mutants, and propose the mechanism of DENV suppression by PCPBAe1. Using hydrogen-deuterium exchange mass spectrometry (HDXMS), site-directed mutagenesis, and ELISA, we mapped the interaction interface between PCPBAe1 and the DENV-2 E protein. Collectively, our studies provide the opportunity (i) to design antibodies or inhibitors against mosquito carboxypeptidases and (ii) to develop novel strategies to control DENV propagation in the mosquito vector.

\section{Results}

\section{Sequence analysis of the PCPBAe1 gene}

Tham et al reported a putative procarboxypeptidase B1 (annotated CPB1), from Ae. aegypti mosquito midgut $(6,7)$. Here, we have adopted the conventional naming system to differentiate the fulllength procarboxypeptidase (FL-PCPBAe1) from the mature peptidase domain alone (CPBAe1) with "Ae" denoting the Ae. aegypti's origin of the gene. Besides, the PCPBAe1 amino acid residue numbering used here adopts the conventional numbering system of carboxypeptidases (10, 11, 20). Based on sequence analyses (Fig 1A), PCPBAe1 is expressed as a preproprotein, with a signal peptide $\left(\right.$ Met $^{*}-\mathrm{Ala}^{*}$ ), an N-terminal pro-region (Ala ${ }^{1 \mathrm{~A}}-\mathrm{Asp}^{75 \mathrm{~A}}$; "A" to indicate the activation unit or pro-region), and a $C$-terminal mature peptidase domain (Asp ${ }^{7}-\mathrm{Phe}^{305}$ ) (CPBAe1). There is an activation loop between $\mathrm{Asp}^{75 \mathrm{~A}}$ and $\mathrm{Asp}^{7}$, with a primary trypsin cleavage site located between the scissile peptide bond, $\operatorname{Arg}^{6}-\mathrm{Asp}^{7}$ (Fig 1A). A unique feature of PCPBAe1 is the missing $3_{10}$-helix insertion, which is typical to $\operatorname{CPBs}(20,21)$. From our analysis, we observe that this feature may only be unique to mammalian B-type MCPs and not insect B-type MCPs like CPBAe1 and CPBHz (Fig 1B).

\section{Structure of PCPBAe1 and the pro-region auto-inhibition mechanism}

Crystal structure of PCPBAe1 was determined to $2.08-\AA$ resolution (Fig 2) 7EQX. PCPBAe1 elutes as a monomer in solution as revealed by size-exclusion chromatography and dynamic light scattering (Fig $\mathrm{S} 1 \mathrm{~A}-\mathrm{C}$ ). However, the structure consists of two molecules in the asymmetric unit of the crystal. Each molecule consists of 394 amino acid residues (Glu ${ }^{5 \mathrm{~A}}-\mathrm{Phe}^{305}$ ) (Fig 2). The activation loop ( $\mathrm{Asp}^{75 \mathrm{~A}}$ $\mathrm{Asp}^{7}$ ) that connects the pro-region to the mature peptidase domain is disordered and could not be modeled in our structure. The pro-region adopts an open-sandwich antiparallel- $\alpha$ / antiparallel$\beta$-fold, consisting of two $\alpha$-helices and four $\beta$-strands. The mature peptidase domain contains a zinc-coordinated active site triad and the substrate-binding pocket and displays a core of eight twisted $\beta$-sheets surrounded by eight $\alpha$-helices (Fig 2).

The globular N-terminal pro-region occupies the wide active site cleft of the mature peptidase domain, mediated via interactions with residues from the substrate-binding pocket of the peptidase
(A)

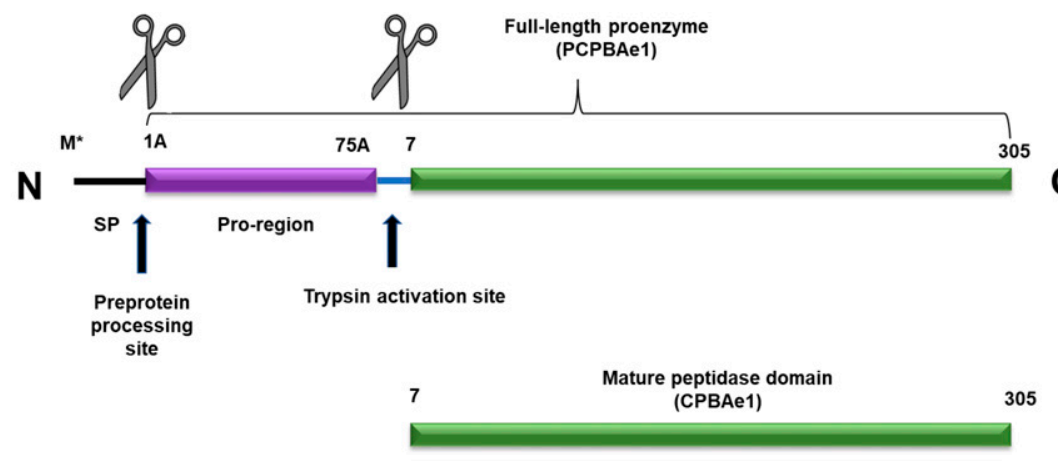

(B)

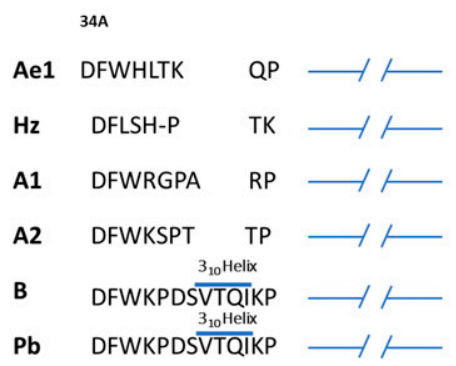

$66 \mathrm{~A} \quad \beta 4 \quad \alpha 3$

GL

GLHYQEVI

VEGTLAP

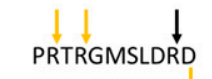

B4 103 GITYRTHADDVKR $\overline{Q L E}$ GIRYTIMIEDVQLLLI $\frac{\beta 4}{A Y S I M I E D V Q V L L D}$ $\alpha_{\alpha 3}$ DQLIEMQRMSSFTRTAGRQL 作 $\frac{\beta 4}{\text { ELQYEVLINNLR R SVLFAQ }}$ ELQYEVLINNLRSVLEAQ
Figure 1. Domain architecture and sequence analysis of full-length PCPBAe1.

(A) Sequence architecture of full-length Ae. aegypti procarboxypeptidase B1 (PCPBAe1) showing the signal peptide (SP) $\left(M^{*}-\operatorname{Arg}^{1 A}\right), N$-terminal pro-region $\left(\operatorname{Arg}^{1 A}-A s p^{75 A}\right)$, and a C-terminal mature peptidase domain $\left(\mathrm{Asp}^{7}-\mathrm{Phe}^{305}\right)$ separated from the pro-region by an activation loop ( Asp $^{75 \mathrm{~A}}$-Asp $\left.{ }^{7}\right)$. SP and pro-region cleavage sites are indicated with black arrows. (B) Comparison of the sequences of regions in the activation segments of PCP that interact with the enzyme moiety. The sequences correspond, from top to bottom, to PCPBAe1 (Ae1), procarboxypeptidase B from $\mathrm{H}$. zea $(P C P B H z)(H z)$, the $A 1, A 2$, and $B$ forms of procarboxypeptidases from bovine and porcine $(\mathrm{Pb})$. The regions around residue 40 (left) and the C-terminus of the activation segment (right) are shown; the first residues of every sequence segment (34A and 66A) are indicated; " $A$ " refers to residues belonging to the activation segment (pro-region). Strand $\beta 4$ and helix $\alpha 3$ are present in all five sequences, whereas only the $B$ form from bovine and porcine possesses a $3_{10}$ helix turn which is missing in PCPBAe1 and CPBHz. Black and yellow arrows indicate the primary and secondary trypsin cleavage sites, respectively. 

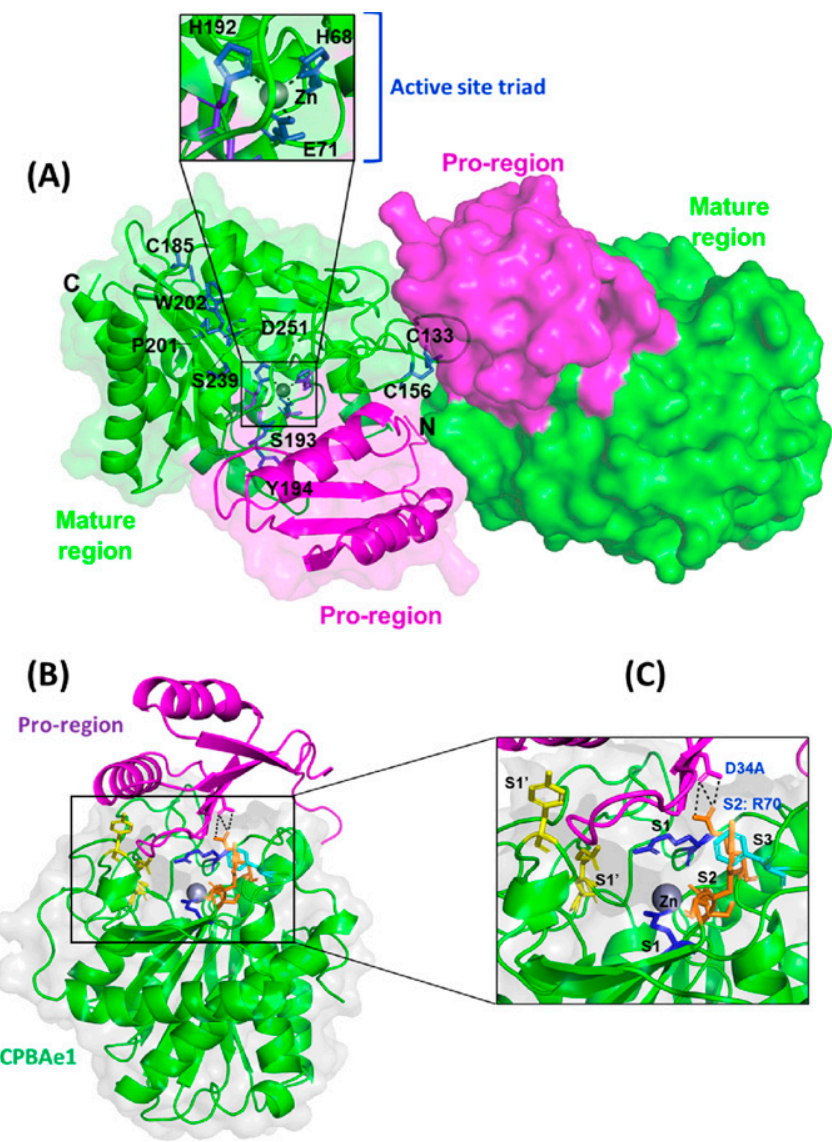

Figure 2. Crystal structure of PCPBAe1 and the autoinhibition mechanism by the pro-region.

(A) Overall structure of PCPBAe1 7EQX. There are two molecules in the asymmetric unit of the crystal. Pro-region (magenta) and mature peptidase domain (green) are indicated for each molecule in cartoon and surface representations. Close-up view of the active site pocket (inset) highlights the active site triad $\mathrm{H}^{68}, \mathrm{E}^{71}$ and $\mathrm{H}^{192}$, coordinated by a zinc atom (grey spheres). A disulfide bond is shown at $\left(\mathrm{C}^{133}-\mathrm{C}^{156}\right)$, whereas a free $\mathrm{C}^{185}$ is also shown. Two cis-peptide bonds $\left(\mathrm{S}^{193}-\mathrm{Y}^{194}\right.$ and $\left.\mathrm{P}^{201}-\mathrm{W}^{202}\right)$ are shown with green. Important substrate specificity residues $D^{251}$ and $S^{239}$ are also indicated. (B) A single molecule of PCPBAe1 showing the auto-inhibition mechanism. (C) Close-up view of the substrate binding pocket of the mature enzyme with the subsites $S_{1}{ }^{\prime}$ $\left(\mathrm{Asp}^{251}, \mathrm{Tyr}^{244}, \mathrm{Asn}^{139}\right.$, and $\mathrm{Arg}^{140}$ ) in yellow; $\mathrm{S}_{1}\left(\mathrm{Glu}^{267}\right.$ and $\left.\mathrm{Arg}^{123}\right)$ in blue; $\mathrm{S}_{2}\left(\mathrm{Arg}^{70}\right.$, $\mathrm{Glu}^{94}$, $\mathrm{Ser}^{193}$, Tyr $^{194}$, and $\left.\mathrm{Gly}^{195}\right)$ in light orange; and $\mathrm{S}_{3}\left(\mathrm{Phe}^{276}\right)$ in dark orange are shown as sticks. Pro-region of PCPBAe1 ( $\mathrm{Asp}^{34 \mathrm{~A}}$ ) forms hydrogen bonds with the $\mathrm{S}_{2}$ subsite $\left(\mathrm{Arg}^{70}\right)$ (black dashed lines). Zinc atom is in grey.

region (Fig 2B). Residues $\mathrm{Asp}^{34 \mathrm{~A}}$ and $\mathrm{Ph} \mathrm{e}^{35 \mathrm{~A}}$ from the pro-region make contacts with the $\mathrm{S}_{2}$ subsite residue $\operatorname{Arg}^{70}$ (Fig 2C); this interaction is crucial for blocking small-peptide substrates from contacting the zinc atom, and thus maintain PCPBAe1 in its inactive form. In particular, the double hydrogen bond between $\mathrm{Asp}^{34 \mathrm{~A}}$ and $\mathrm{Arg}^{70}$ might explain how PCPBAe1 remains completely inactive as compared to mammalian PCPA (22). Although other contacts are outside the region of substrate-binding residues, they are also close enough to block substrate binding. For example, Asn ${ }^{30 A}$ contacts $\mathrm{Gln}^{164}$, which blocks the $\mathrm{S}_{1}$ subsite residue $\mathrm{Asp}^{168}$. Similarly, $\mathrm{Arg}^{47 \mathrm{~A}}$ interacts with $\mathrm{Gly}^{274}$, limiting access to the $S_{3}$ subsite Phe ${ }^{276}$ (Fig $2 \mathrm{C}$ ). Thus, by covering the active site and substrate-binding pocket, the pro-region regulates the activity of the enzyme and therefore, only the removal of the proregion will allow access to substrate or inhibitor binding.

\section{Active site and substrate-binding pocket of the mature peptidase} domain, CPBAe1

The mature CPBAe1 was generated by trypsin limited proteolysis of the full-length PCPBAe1, and further purified using size-exclusion and ionexchange chromatography (Fig S1D). We tested the activities of the wild type (WT), single and double mutants of CPBAe1 (Asp ${ }^{251} \mathrm{Glu}$, Ser ${ }^{239} \mathrm{Gly}$, and $\mathrm{Asp}^{251} \mathrm{Glu}+\mathrm{Ser}^{239} \mathrm{Gly}$ ) using three carboxypeptidase substrates: Hippuryl-L-Arginine (Hip-L-Arg), Hip-L-Lys, and Hip-L-Phe (Table 1). The inactive enzyme PCPBAe1 was used as a negative control. CPBAe1 preferentially hydrolyzed Hip-L-Arg over Hip-L-Lys and displayed undetectable activity against Hip-L-Phe, which is a CPA-specific substrate $(5,23)$ (Table 1). The proenzyme PCPBAe1 showed no intrinsic activity against any of the substrates tested; this is contrary to the CPA family, which exhibit intrinsic activity against small-peptide substrates (24).

One zinc atom is present in each molecule of the structure and forms pentahedral coordination with the catalytic residues: $\mathrm{His}^{68} \mathrm{~N}^{\delta 1}$, $\mathrm{His}^{192} \mathrm{~N}^{\delta 1}, \mathrm{Glu}^{71} \mathrm{O}^{\varepsilon 1}$, and $\mathrm{O}^{\varepsilon 1}$ atoms and a water molecule. CPBAe1 contains one disulfide bond (Cys ${ }^{133}-\mathrm{Cys}^{156}$ ), which is the only conserved disulfide bond present in MCPs $(10,11,20)$ (Figs 2A and S2). However, CPBAe1 has an additional free cysteine $\left(\mathrm{Cys}^{185}\right.$ ) buried near the zinc-binding motif (Figs 2A and S2). Currently, there is no function ascribed to this cysteine residue. Moreover, CPBAe1 displays two of the three cis-peptide bonds (Ser ${ }^{193}-\operatorname{Tyr}^{194}$ and $\mathrm{PrO}^{201}-\operatorname{Trp}^{202}$ ) present in mammalian MCPS (Figs $2 \mathrm{~A}$ and S2) $(10,11,20,23,25)$.

Combined with literature (10), we find that the key residues in the substrate-binding pocket of CPBAe1 include Asp ${ }^{251}$, Ala ${ }^{246}$, Try $^{244}$, $\mathrm{Ser}^{239}, \mathrm{Gly}^{203}, \mathrm{Glu}^{170}, \operatorname{Arg}^{140}$, and $\operatorname{Arg}^{120}$ (Fig 2C). According to Titani et al, $\operatorname{Tyr}^{244}$ and $\mathrm{Arg}^{140}$ in CPBs anchor the terminal carboxyl group of substrates and prime it for hydrolysis (26). Other relevant residues for catalysis, such as $\mathrm{Glu}^{170}$ and $\mathrm{Arg}^{120}$, occupy similar positions in CPBAe1 compared with other MCP enzymes (Fig S2). Gly ${ }^{203}$ of CPBAe1 is also conserved in both pancreatic and insect CPBs and CPAS, except for human pancreatic CPBh and porcine CPBp, where the residue in the equivalent position is occupied by Ser (Fig S2). The presence of $\mathrm{Ser}^{239}$ in CPBAe1, which is conserved in Helicoverpa zea $\mathrm{CPBHz}$ and other mosquito $\mathrm{CPBS}$, narrows the $\mathrm{S}_{1}{ }^{\prime}$ specificity pocket and affects the substrate-binding efficiency of the enzyme (Fig $3 \mathrm{~A}$ and C). Of particular interest, $\mathrm{Asp}^{251}$ at the bottom of the substrate specificity pocket in CPBAe1 (Fig 2A) is also critical for Arg-substrate hydrolysis (Table 1). Thus, our kinetics studies showed that the substrate specificity is dictated by amino acid residue combinations in positions 251 and 239 (Table 1).

So far, the only insect digestive CPB structure available is that of $\mathrm{CPBHz}$ from $\mathrm{H}$. zea (11). CPBHz does not hydrolyze Arg-substrates, and has highly specific interaction towards Lys-substrates; this property was speculated to be dictated by the presence of Glu $\mathrm{u}^{255}$ (Asp ${ }^{251}$ in CPBAe1) in place of Asp in $\mathrm{CPBHz}$ (11) (Fig S2). From our site-directed mutagenesis experiments, $\mathrm{Asp}^{251} \mathrm{Glu}$ mutation abrogated Arg-substrate hydrolysis completely in CPBAe1, whereas Lyssubstrate hydrolysis remained unaffected (Table 1). Interestingly, the double mutant $\mathrm{Asp}^{251} \mathrm{Glu}$ and $\mathrm{Ser}^{239} \mathrm{Gly}$ restored Arg-substrate hydrolysis by CPBAe1, which was comparable to WT CPBAe1. Besides, $\mathrm{Asp}^{251}$ and $\mathrm{Ser}^{239} \mathrm{Gly}$ further enhance Arg-substrate hydrolysis $>30$ fold as compared with WT CPBAe1 (Table 1). To further understand these substrate specificities, we compared Arg-hydrolyzing and non-Arg-hydrolyzing carboxypeptidases (Fig 3A). A detailed analysis 
Table 1. Substrate-hydrolysis of WT and mutant CPBAe1.

\begin{tabular}{l|l|lllll}
\hline Enzymes/Substrates & $\mathbf{2 5 1}$ & $\mathbf{2 3 9}$ & Hip-L-Arg & Hip-L-Lys & Hip-L-Phe & PCPBAe1 \\
\hline & Asp & Ser & $17,000.34$ & $11,600.41$ & 0.00 & 0.00 \\
\cline { 2 - 7 } & Glu & Ser & 0.00 & $11,443.22$ & 0.00 & 0.00 \\
\cline { 2 - 8 } & Asp & Gly & $25,012.10$ & $11,216.91$ & 0.00 & 0.00 \\
\cline { 2 - 8 } & Glu & Gly & $15,971.50$ & $11,930.00$ & 0.00 & 0.00 \\
\hline
\end{tabular}

SEA, specific enzyme activity; 251 and 239 residues positions of WT and mutant CPBAe1.

of the substrate-binding pocket of CPBAe1 and CPBHz superimposed onto the duck CPD-GEMSA (arginine analogue carboxypeptidase inhibitor) complex (PDB: 1H8L) (27) revealed an apparent difference in the substrate-binding pockets of these enzymes (Fig $3 \mathrm{~A})$. Moreover, $\mathrm{Ala}^{246}$, a key residue in the substrate-binding pocket of CPBAe1, is conserved among MCPs except in $\mathrm{CPBHz}$, which has an lle at this position (Fig S2).

\section{Comparison of PCPBAe1 structure with other procarboxypeptidase structures}

A DALI structural homology search identified structural similarities among MCP proenzymes of the A/B subfamily of both insect and mammalian origins (Fig S3A-C and Table S1). Most of the secondary structures aligned well with the PCPBAe1 structure (Fig S3A-C). The nearest structural homolog of PCPBAe1 is the insect proCPA from Helicoverpa armigera (PCPAHa) (Fig S3A). PCPAHa aligns well with PCPBAe1 (PDB: 1JQG, 39\% sequence identity, RMSD $1.4 \AA$ for 394 C $\alpha$ atoms) over the mammalian counterpart (PDB: 1KWM, 36\% sequence identity, RMSD $1.6 \AA$ for 394 C $\alpha$ atoms) (Table S1).
Overall, most of the secondary structures of PCPBAe1 are wellconserved. However, an apparent rotation of $\sim 8-10^{\circ}$ is observed in the pro-region, perhaps because of the less-conserved nature of the residues in the pro-region ( $20 \%$ sequence identity) compared with that of the mature peptidase domain ( 40\% sequence identity) of the homologs (PDBs: 1JQG, 1KWM, and 1NSA) (Figs S2 and S3A-C and Table S1).

\section{PCPBAe1 directly interacts with DENV-2 infectious virion, VLP, and $E$ protein}

Previous work suggested that PCPBAe1 inhibited DENV-2 release from the mosquito midgut cells, by binding to and hijacking the viral E protein/virion, thereby preventing the encapsulation of newly formed nucleocapsids or preventing virion maturation $(6,7)$. Interestingly, PCPBAe1 overexpression affects mature DENV-2 release from the mosquito $\mathrm{C} 6 / 36$ cell line, but not from mammalian Vero cells (7). Here, we conducted ELISA assays using recombinant PCPBAe1 purified from Escherichia coli (ECP) and insect baculoviral expression systems (ICP) (Fig S1A and C). ECP and ICP were

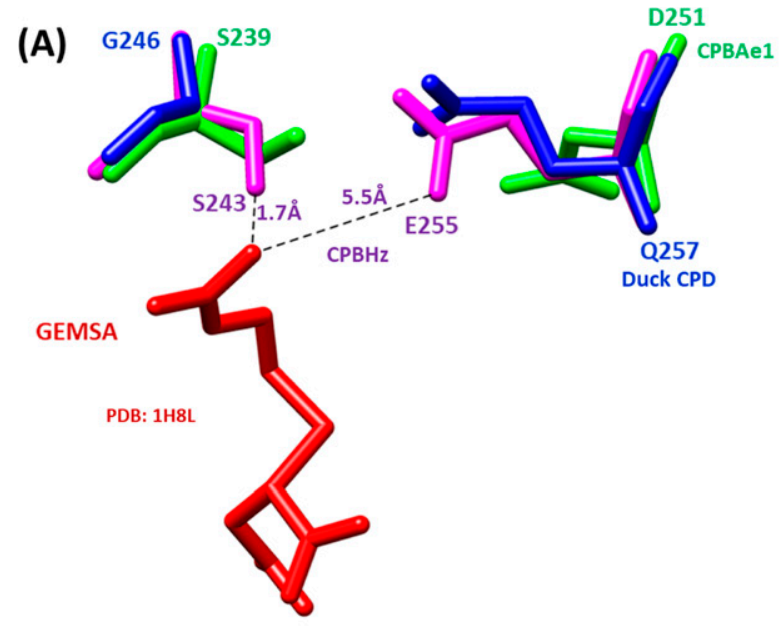

(B)

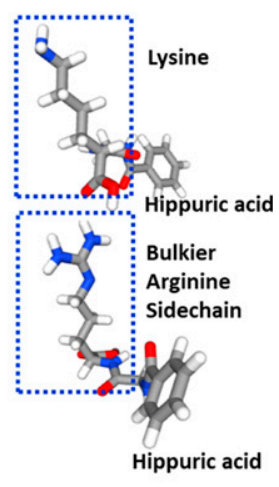

Figure 3. Structural comparison of the substratebinding pocket of CPBAe1 and $\mathrm{CPBHz}$ with duck CPD-GEMSA complex (Protein Data Bank [PDB]:1H8L). (A) The substrate-binding pocket is governed by Asp ${ }^{251}$ (for CPBAe1), $\mathrm{Glu}^{255}$ (for $\mathrm{CPBHz}$ ), and $\mathrm{Gln}^{257}$ (for CPD) and $\operatorname{Ser}^{239}$ (for CPBAe1), $\operatorname{Ser}^{243}$ (for CPBHz) and $\mathrm{Gly}^{246}$ (for CPD). The distance between GEMSA (red) and $\mathrm{CPBHz}$ are indicated. (B) Chemical structures of hippuryl-LArginine and hippuryl-L-Lysine showing the side chains. (c) Schematics showing the proposed substrate-binding architecture dictated by different combinations of amino acid residues.
(C)
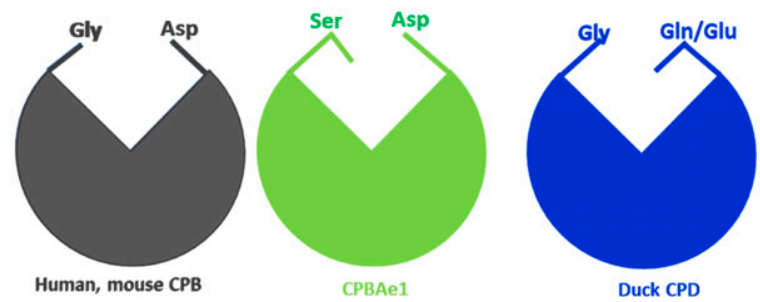

Duck CPD

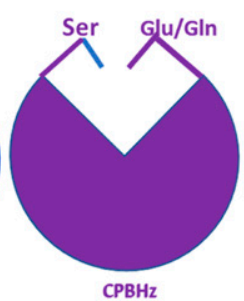


incubated with DENV-2 E protein obtained from insect and mammalian expression systems (Fig S1F), VLPS purified from mammalian cells, and infectious virions of different DENV serotypes from the mosquito C6/36 cell line (Fig 4A). Different sources of proteins/ VLPS/virions were used as a way to possibly uncover the mechanism behind the different effects of PCPBAe1 on DENV-2 release from different cell lines (7).

ECP and ICP interacted with insect- and mammalian cell-derived viral coat antigens/virions (Fig 4A). ICP showed marginally higher interaction with all the viral antigens/virions ( 10-13-fold higher) as compared with ECP (Fig 4A). The reason for this higher interaction is unclear. Gel electrophoresis analysis showed that both ICP and ECP have similar molecular weights (Fig S1A and C). Hence, the difference in interaction is unlikely due to any post-translational modifications on ICP. We also noted that PCPBAe1 had a stronger interaction ( 25-30fold higher) with the insect-purified $E$ protein than the mammalianpurified E protein/VLP (Fig 4A). This interaction disparity might be due to the source of the viral antigens, which likely have variations in posttranslational modifications on the E glycoproteins. There are two $\mathrm{N}$ glycosylation sites $\left(\mathrm{Asn}^{67}\right.$ and $A s n^{153}$ ) on the DENV E protein (28), and the differences in the compositions of glycans between insect- and mammalian-purified glycoproteins (29) might play a role in the interaction disparities. The interaction with the peptidase domain (CPBAe1) or the pro-region (ProCP) alone (Fig 4B) (purified from E. coli; Fig S1D and E), led to a reduction in binding with DENV ( $>50$-fold lower) suggesting that the full-length-PCPBAe1 is required for interaction. There was no interaction between PCPBAe1 and VLPS of chikungunya (CHIKV) and Mayaro (MAYV) viruses (two alphaviruses that are also transmitted by Aedes mosquitoes) (data not shown).

\section{Mapping the interaction regions of $\mathrm{PCPBAe} 1$ with the $\mathrm{E}$ protein using HDXMS}

We used HDXMS and high-resolution structures to map the interacting regions of PCPBAe1 and the DENV-2 E protein (Fig 5A-C). We observed that the $\mathrm{N}$-terminal pro-region of PCPBAe1 $\mathrm{Ala}^{1 \mathrm{~A}}-\mathrm{Asp}^{75 \mathrm{~A}}$ (notably region
$\mathrm{Tyr}^{7 \mathrm{~A}}-\mathrm{Ala}^{21 \mathrm{~A}}$ ) showed a single highest deuterium uptake than the peptidase domain $\mathrm{Asp}^{7}-\mathrm{Phe}^{295}$ which exhibited multiple increased deuterium uptake (peptide clusters 171-182, 206-218 and 226-252) upon binding to $\mathrm{E}$ protein (Fig $5 \mathrm{~A}$ and C). Only one locus (the linker between the pro-region and the peptidase domain $\left[\right.$ Pro $^{82 \mathrm{~A}}-$ Leu $\left.^{4}\right]$ ) of PCPBAe1 showed significant protection against deuterium exchange in the PCPBAe1:E protein complex. Consequently, these identified sites were mutated to alanine or deleted from loop regions in each peptide and showed varying levels of reduction in the interaction as compared with wild-type PCPBAe1 (Fig 6). The extreme N-terminal exposed Asp ${ }^{18 \mathrm{~A}}$ and $\mathrm{Glu}^{19 \mathrm{~A}}$ deletion caused the most dramatic reduction in the interaction ( 50 -fold lower), followed by the charged residues mutations to alanine in the buried linker region ( $\mathrm{PrO}^{82 \mathrm{~A}}$ - $\mathrm{Leu}^{4} ; \mathrm{Glu}^{85}, \mathrm{Arg}^{87}$, and $\mathrm{Arg}^{89}$-AlaAlaAla) which showed moderate reduction in interaction ( 20-fold) (Fig 6A). Deletion of the entire linker loop resulted in inclusion body formation and could not be purified and tested. The extreme $\mathrm{C}$-terminal residues mutations $\mathrm{Glu}^{168}, \mathrm{Glu}^{170}, \mathrm{Arg}^{172}$-AlaAlaAla (peptide $\mathrm{Gly}^{162}-\mathrm{Arg}^{172}$ ), and $\mathrm{Val}^{300}$-Phe ${ }^{305}$ deletion did not impact the interaction (Fig 6A).

PCPBAe1 primarily targets the exposed flexible loop regions of the $E$ protein domains I/II; although, the interaction does not include the fusion loop (Asp ${ }^{98}$-Gly ${ }^{109}$ ) (Fig 5B and C). The peptide regions of E protein domains I/II $\left(\right.$ Lys $^{64}-\mathrm{Glu}^{84}$, Val $^{238}-\mathrm{Val}^{252}$, Leu ${ }^{278}-$ Leu $\left.^{287}\right)$ showed decreased deuterium exchange, suggesting that these regions are buried upon binding to PCPBAe1. Consequently, we used independent domains (domains I/II, and domain III; purified from E. coli) (Fig S1G and H) as truncated versions of the $E$ protein to localize the importance of these regions in interacting with $\mathrm{PCPBA} 1$. The interaction of the individual domains I/II or III alone with PCPBAe1 is significantly less ( 70-85-fold lower) than that of the full-length $E$ protein, suggesting that the fulllength $\mathrm{E}$ protein is necessary for maximum binding (Fig 6B).

\section{PCPBAe1 binds to all DENV serotypes and 67NTT-67QTV and N153Q mutants}

Sequence alignment of all four DENV serotype E proteins (Fig 7A) revealed some residue conservations among the buried peptides

\section{Insect purified E protein}

$\square$ Mammalian purified $E$ protein

- Mammalian purified VLP

(A)

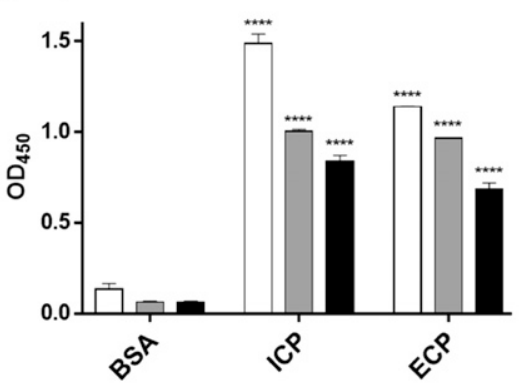

(B)

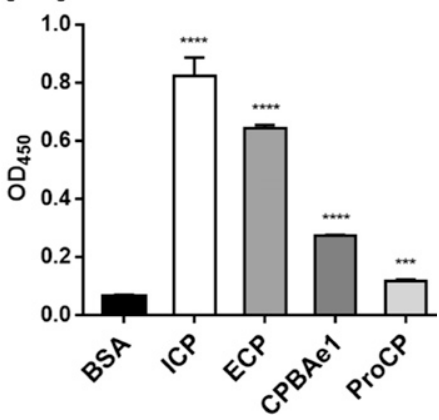

Figure 4. PCPBAe1, CPBAe1, and ProCP interaction with DENV-2 E protein/virus-like particles or infectious virion.

(A, B) All the interaction studies (panel A, B) were performed by ELISA in triplicates. (A) Insect cellpurified PCPBAe1 (ICP) and E. coli purified PCPBAe1 (ECP) interaction with insect cell purified $E$ protein and mammalian HEK purified E protein/virus-like particles. (B) PCPBAe1 (ECP/ICP), mature peptidase domain (CPBAe1) and pro-region (ProCP) interaction with DENV-2 infectious virion. CPBAe1 and ProCP were produced from the $E$. coli expression system. Data were analyzed by independent unpaired t-test between two sets of data (control and treated samples), and by two-way ANOVA for multiple groups of data using GraphPad Prism version 7.00. Results are shown as mean \pm S.D. ${ }^{*} P<0.05,{ }^{* *} P<0.01,{ }^{* * *} P<0.001$, and $\star * * * P<0.0001$. 
(A) Fig. 5

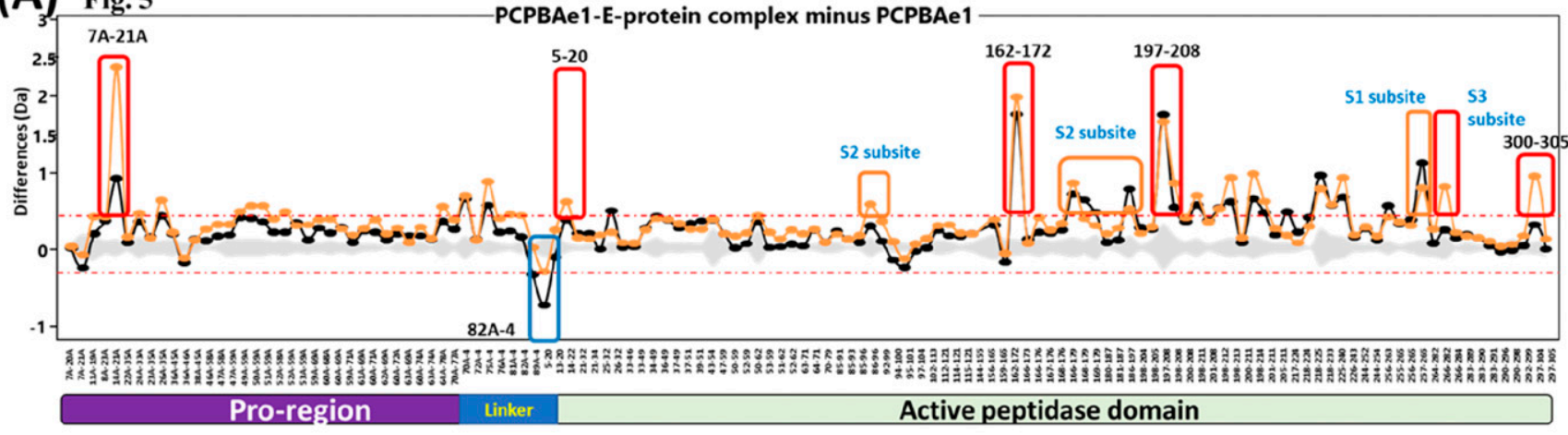

(B)

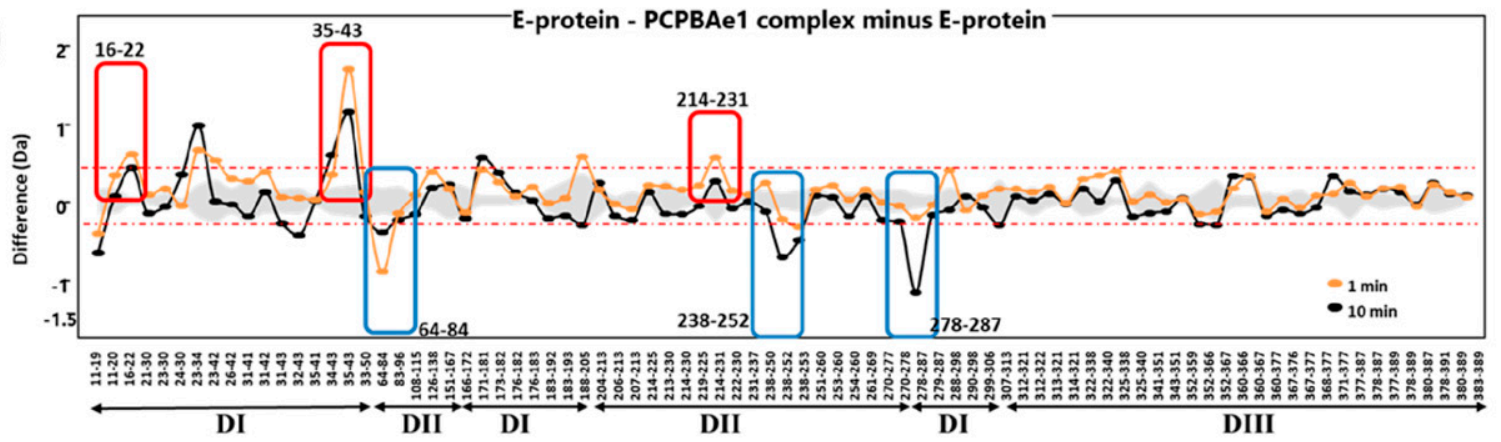

(C)

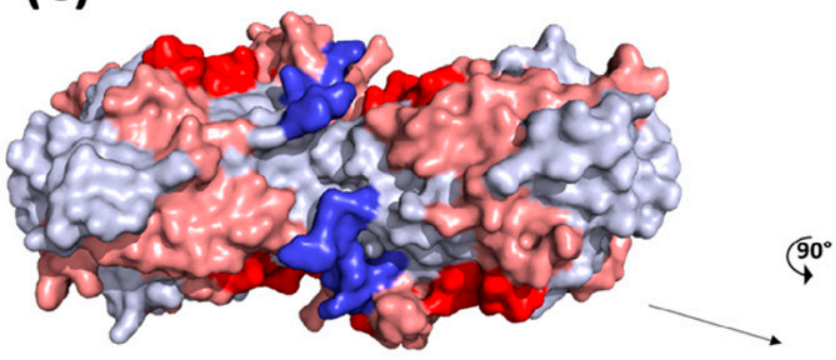

PCPBAe1
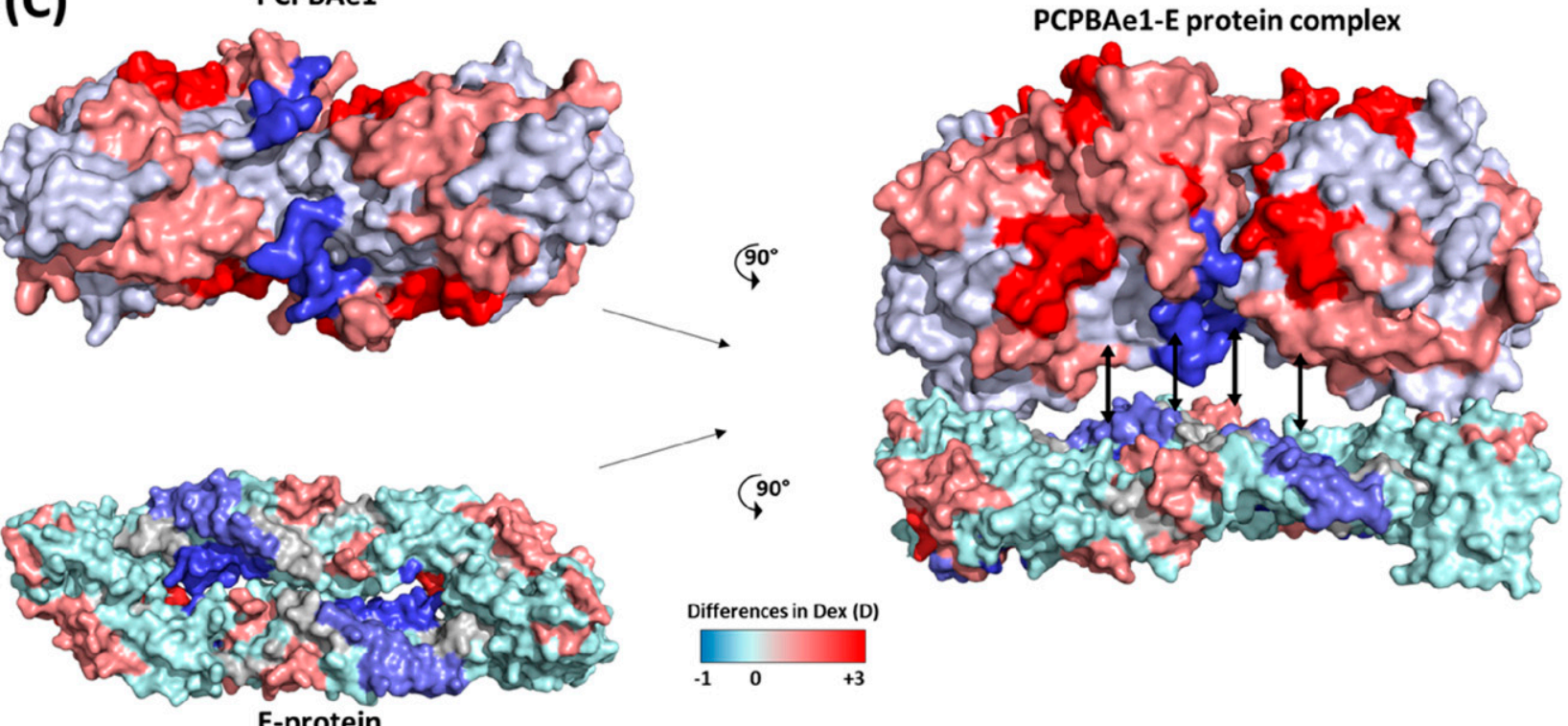

Differences in Dex (D)

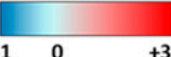

E-protein

Figure 5. Effect of E protein-PCPBAe1 binding on each protein and proposed binding mode.

(A) Plot depicting the differences in deuterium exchange between E protein bound and free PCPBAe1 for various pepsin digested fragments at 1 min (orange) and 10 min (black) labeling timescales. Residue numbers spanning the $\mathrm{N}$ - to C termini of PCPBAe1 are indicated, as per the pro-region and peptidase domains. (B) Difference plot of differences in deuterium exchange (y-axis) between PCPBAe1-bound and free E protein for various pepsin digest fragments of $E$ protein ( $x$-axis). Residue numbers of various peptides with domain (I, II, and III) organization is shown. Peptide Ala ${ }^{35}$-Asp ${ }^{43}$ spanning domains I showed large-scale increased deuterium exchange in the complex. (A, B) Positive differences indicate increased deuterium exchange and negative values indicate decreased deuterium exchange in E protein bound to PCPBAe1 as compared with (A) PCPBAe1 alone or (B) E protein alone. Blue boxes highlight peptides of E protein or PCPBAe1 showing significant "protection" against deuterium uptake in the peptides in PCPBAe1:E protein complex. Each value is an average of three independent labeling measurements and the standard deviations are in grey. $A$ threshold of $\pm 0.3 \mathrm{D}$ is considered as significance cut-off and indicated by red-dashed lines. (C) Differences in deuterium exchange at 10 min labeling time are mapped on to the crystal structures of PCPBAe1 (PDB: 7EQX) (solved in this study) (left panel, top) and E protein (left panel, bottom) dimer (PDB: 10AN), shown in surface representation. Difference maps are colored according to the key with regions showing decreased deuterium exchange in shades of blue and increased deuterium exchange in shades of red. A surface representation model of PCPBAe1-E protein complex (right panel) showing the interaction interface is shown, with the predicted regions of interaction indicated by double-headed arrows. 
(A)

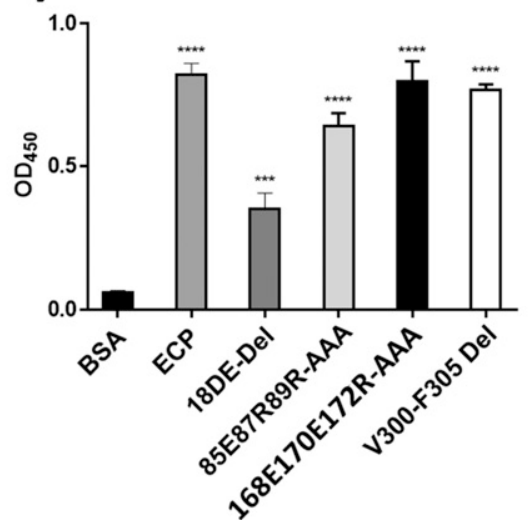

(B)

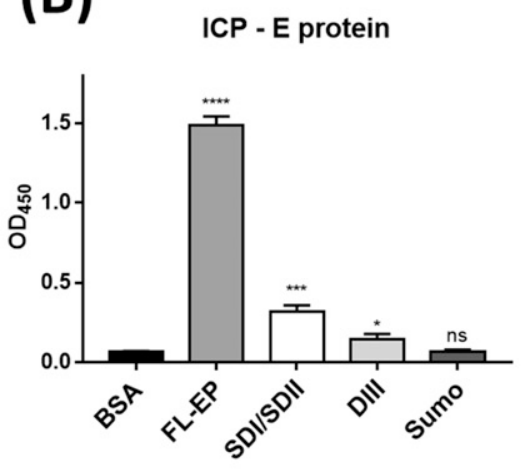

(C)

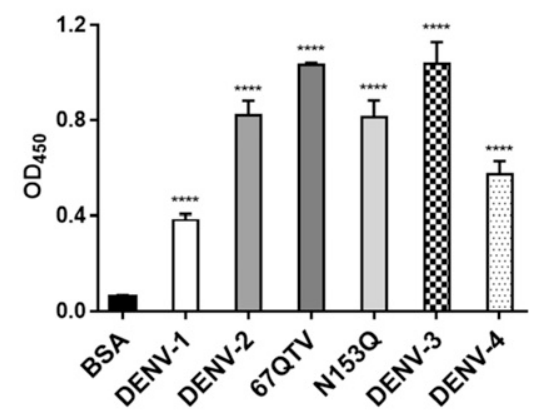

Figure 6. WT PCPBAe1, mutant PCPBAe1, DENV-E protein, and infectious virion interaction.

$(\mathbf{A}, \mathbf{B}, \mathbf{C})$ All the interaction studies (panels A, B, C) were performed by ELISA in triplicates. (A) Wild-type and mutants of ECP interaction with DENV-2 infectious virion. (B) Insect-purified full-length E protein and E. coli purified E protein domains (DI/II) and (DIII) interaction with ICP. (C) DENV serotypes and mutants interaction with ECP. Data were analyzed by independent unpaired t-test between two sets of data (control and treated samples), and by two-way ANOVA for multiple groups of data using GraphPad Prism version 7.00. Results are shown as mean \pm S.D. ${ }^{\star} P<0.05,{ }^{* \star} P<0.01,{ }^{* \star *} P<0.001$, and ${ }^{* \star * \star} P<0.0001$.

$\left(\right.$ Lys $^{64}-\mathrm{Glu}^{84}, \mathrm{Val}^{238}-\mathrm{Val}^{252}$, and $\left.\mathrm{Leu}^{278}-\mathrm{Leu}^{287}\right)$ and among the peptides that exhibited increased deuterium exchange (Ser ${ }^{16}$ $\mathrm{Asp}^{22}, \mathrm{Ala}^{35-43}$, and Leu ${ }^{214}-\operatorname{Trp}^{231}$ ) (Fig 5B). ELISA experiments with the four DENV serotypes showed different levels of interaction in the following decreasing order: DENV-3 > DENV-2 > DENV-4 > DENV-1 (Fig 6C). From the sequence alignment, differences in key amino acid residues of the $\mathrm{E}$ protein interacting regions (Fig 7B) may be responsible for the observed differences in affinity (Fig 6C). A protein-wide overview of the HDXMS data indicated that many regions showed higher deuterium exchange near the $\mathrm{E}$ protein glycosylation site $\mathrm{Asn}^{67}$ and not $\mathrm{Asn}^{153}$. To test the effect of $A s n^{67}$ and $\mathrm{Asn}^{153}$ in the interaction between DENV-2 and PCPBAe1, we generated partially deglycosylated DENV-2 mutants that lack their glycan motif either at N67 (NTT-QTV) or at N153 (N153Q). We then conducted an ELISA assay with 67NTT-67QTV and N153Q mutant DENV-2 virions. From Fig 6C, the 67QTV mutant displayed statistically insignificant stronger interaction with PCPBAe1 than the WT, whereas the N153Q mutant interaction was similar to the WT.

\section{Discussion}

MCPs have adopted a complex function in mosquitoes, with roles in mosquito reproduction (5), enhancing malaria parasite infection (4), but also suppressing DENV replication in the mosquito midgut (7). Here, we determined the first crystal structure of a mosquito MCP, PCPBAe1, and characterized the substrate hydrolysis properties of the mature peptidase domain (CPBAe1). We identified critical differences between CPBAe1 substrate hydrolysis and that of mammalian and other insects MCPS. Furthermore, through a combination of HDXMS, ELISA, and mutational studies, we described and proposed how PCPBAe1 binds to and suppresses DENV infection.

CPBAe1 hydrolyzes Arg- and Lys-substrates, and this substrate specificity is dictated by residues $A_{s p}{ }^{251}$ and $\operatorname{Ser}^{239}$. From the comparison of substrate-binding pocket, the presence of Gly ${ }^{239}$ compensates for Glu/GIn ${ }^{251}$, whereas Asp ${ }^{251}$ compensates for Ser ${ }^{239}$ to accommodate Arg-substrate hydrolysis. Although Asp and Glu have similar physicochemical properties, their side chains affect Arg- and Lys-substrates binding and hydrolysis differently. The Arg side chain of Arg-substrates is bulkier and does not insert easily into the substrate-binding pocket compared with the less bulky Lys side chain. Thus, $\mathrm{Glu}^{251} / \mathrm{Ser}^{239}$ severely restricts the substratebinding pocket and does not allow accommodation of the bulky guanidyl group of peptides containing C-terminal Arg. On the contrary, Asp ${ }^{251} / \mathrm{Gly}^{239}$ allows the side chain of a C-terminal Arg residue of a bound substrate to move away from $\mathrm{Asp}^{251}$, which increases Arg-substrate hydrolysis.

Sequence alignment shows that $\mathrm{Asp}^{251}$ and $\mathrm{Ser}^{239}$ are conserved across mosquito CPBs, and these amino acid residues might be critical for blood digestion and in facilitating egg development (11, 19). This might explain the poor fecundity observed in female mosquitoes when CPB activity was blocked $(11,19)$. Thus, blocking the enzymatic activity of CPBs in mosquitoes could contribute as a mosquito population control strategy.

Solving the structure of PCPBAe1 allowed us to investigate how the full-length PCPBAe1 binds to the DENV-2 E protein to suppress infection. In the absence of PCPBAe1, ingested DENV undergoes normal replication in the midgut (Fig 8A). During blood ingestion, PCPBAe1 becomes activated (CPBAe1) to digest blood proteins. It is possible that the mature CPBAe1, when released into DENV-infected blood in the midgut lumen, binds to mature virions and partially inhibits binding and entry into midgut cells. We surmise that both the full-length-PCPBAe1 and the mature form (CPBAe1) could potentially bind to the virus inside the mosquito (Fig 8B); albeit the full-length-PCPBAe1 might exhibit a stronger inhibition potency against virus propagation due to its ability to bind more strongly ( 50 -fold stronger) to the virus. The weaker interaction of the mature peptidase domain alone in our in vitro assay might be due to the disruption of the putative interaction linker region $\left(\mathrm{Tyr}^{4 \mathrm{~A}}\right.$ $\mathrm{Glu}^{21 \mathrm{~A}}$ ) to produce the active enzyme. The $\mathrm{N}$-terminal peptide region 
Life Science Alliance

(A)

16-22

20

35-43

DENV2E

DENV1E

DENV3E

DENV4E

MRCIGI SNRDFVEGV SGGSWVD VLEHGSCVTTN ARNKP TLDFELIKTEAKQPATLRKYC I EAK L TNTTT

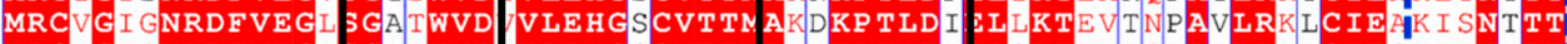

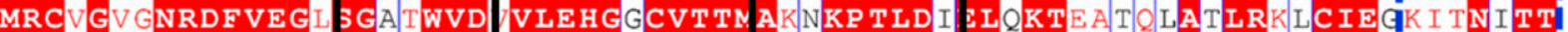

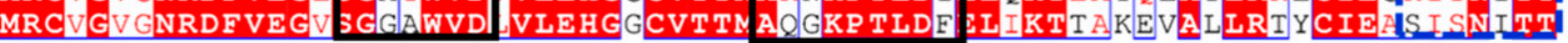

Site 1

64-84

80

Fusion loop

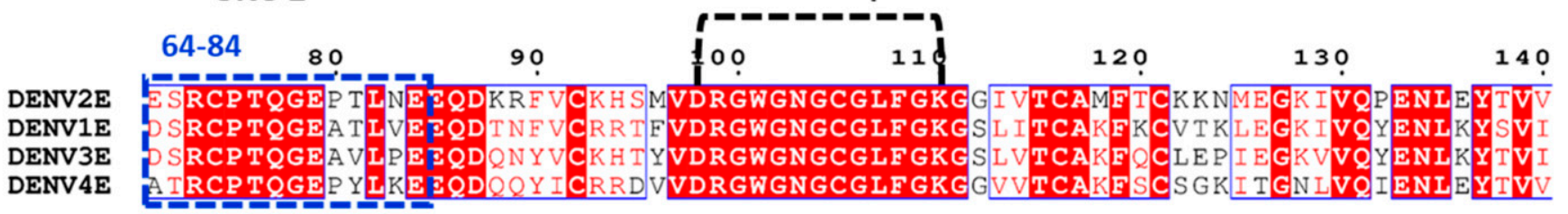

N153

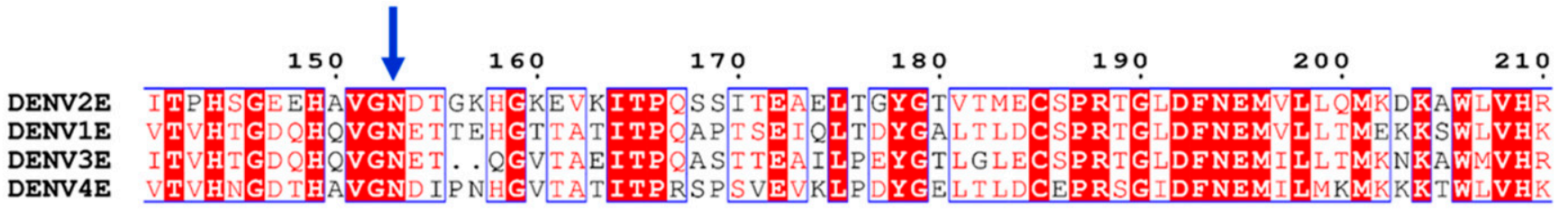

Site 2

214-231

238-252

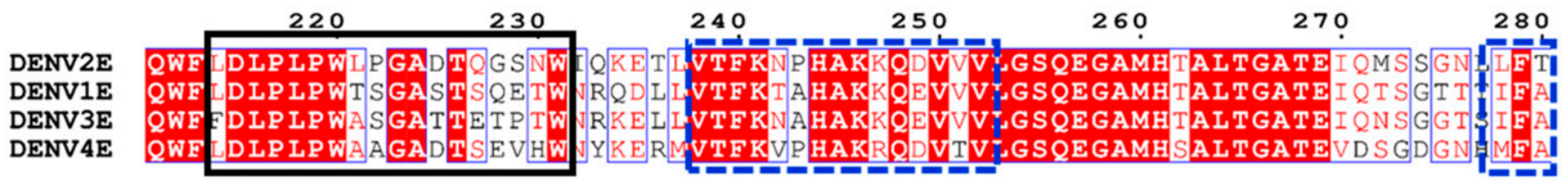

\section{Site 3}

$\begin{array}{lllllll}278-287 & 290 & 300 & 310 & 320 & 330 & 340\end{array}$

DENV2E GHLKCR RMDKL LKGMSYSMCT GKEKVVREI AETQHGTIVIRVQYEGDGSPCKIPFE IMDLEKRHVLGR DENV1E GHLKCRLKMDKL LKGMSYVMCT GSKLEKEVAETQHGTVLVQVKYEGTDAPCKIPF S QDEKGVTQNGR DENV3E GHLKCRLKMDKLELKGMSYAMCTNTFVIKKEVSETQHGTIL I KVEYKGEDAPCK I P F STEDGQGKAHNGR DENV4E LGHLKCKVRMEKLRIKGMSYTMCSGKESIDKEMAETQHGTTVVKVKYEGAG APCKVPIEIRDVNKEKVVGR

\section{(B)}

DENV2E KLTNTTTESRCPTQGEPTLNE

Site 1 DENV1E KISNTTTDSRCPTQGEATLVE

(64-84) DENV3E KITNITTDSRCPTQGEAVLPE

DENV4E SISNITTATRCPTQGEPYLKE
DENV2E VTFKNPHAKKQDVVV

Site 2 DENV1E VTFKTAHAKKQEVVV

(238-252)DENV3E VTFKNAHAKKQEVVV

DENV4E VTFKVPHAKRQDVTV
DENV2E LFTGHLKCRL

Site 3 DENV1E IFAGHLKCRL

(278-287)DENV3E IFAGHLKCRL

DENV4E MFAGHLKCKV

Figure 7. Sequence alignment of $E$ protein from the four DENV serotypes.

(A) The E protein peptides that are buried upon binding to PCPBAe1 are indicated in blue dash boxes spanning protein domains I/II (a.a 64-84, 238-252, and 278-287), whereas peptide regions that underwent increased deuterium exchange are indicated in black boxes spanning E protein domains I/II (a.a 16-22, 35-43, and 214-231). The Fusion loop is also indicated. The two N-glycosylation sites (N67 and N153) are shown with blue arrows. White fonts with red background show conserved residues, whereas red fonts with white background are areas of high similarities. The alignment was done with Clustal Omega and ESPript 3.0. (B) Zoomed-in sequence alignment of E protein regions targeted by PCPBAe1. Important amino acid residue changes are indicated with bold font and yellow highlights. Conserved proline residues are indicated with bold red font. 


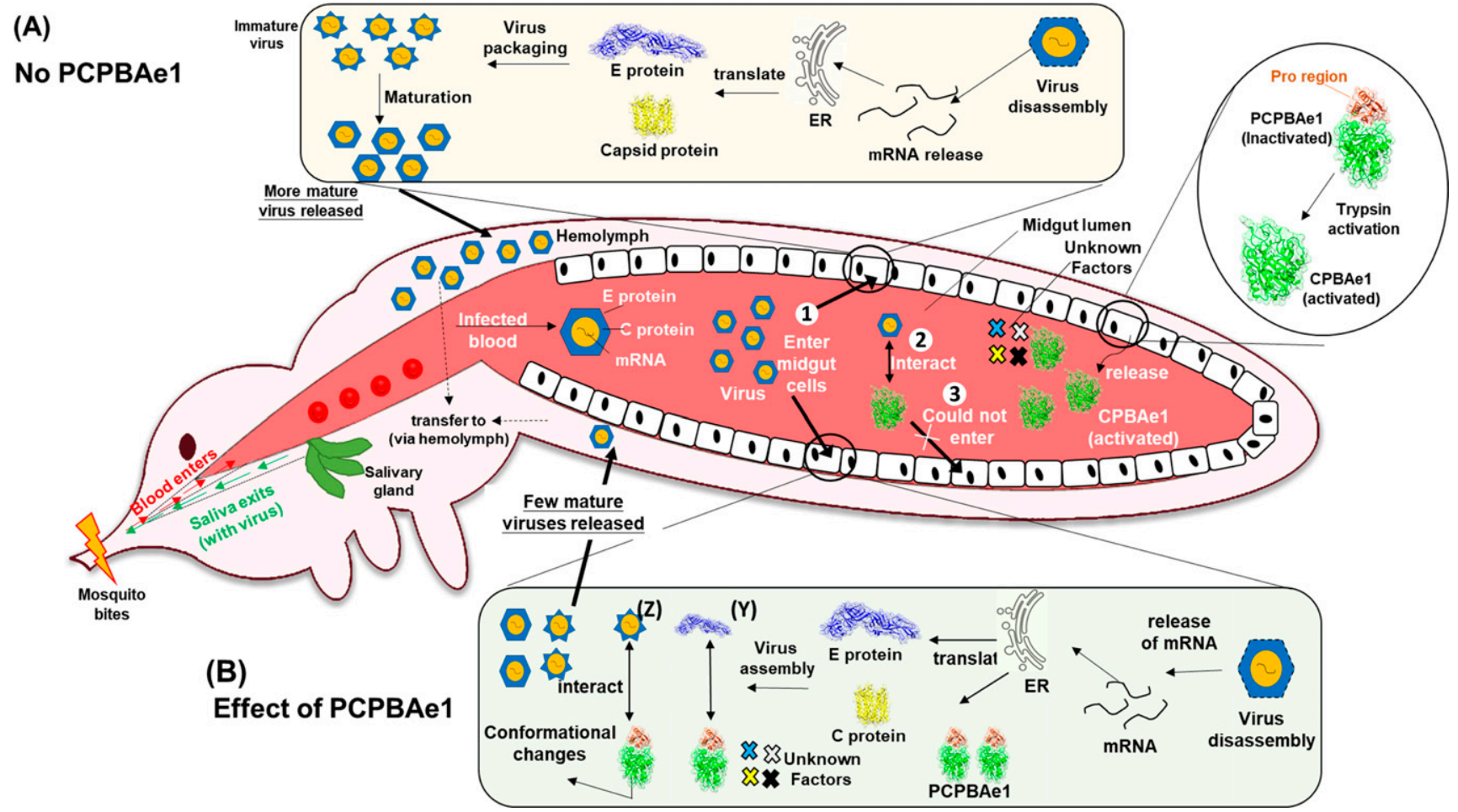

Figure 8. A schematic of the binding and inhibition mechanism of PCPBAe1/CPBAe1 on DENV-2 in the mosquito midgut.

Female mosquito takes in an infected bloodmeal containing DENV. (1) Some DENV particles enters midgut (2) Bloodmeal in the midgut causes overexpression of procarboxypeptidase (PCPBAe1) and activated by trypsin into CPBAe1 (inset, circle), which binds to mature DENV and (3) partially inhibits cellular binding and entry. (A) DENV proliferate as immature viruses, which undergo conformational changes to become mature viruses in PCPBAe1-free cells. Mature viruses are eventually released into the salivary gland and subsequently into the mosquito saliva. (B) In the midgut epithelia, overexpressed PCPBAe1 binds to: (Y) E protein and prevents viral nucleocapsid packing, (Z) immature viruses and prevents conformational changes required for virus maturation. These events lead to a low number of released mature viruses into the hemolymph. Unknown factors possibly exclusive to the mosquito cellular milieu that may augment PCPBAe1 inhibitory activity against DENV are shown as different colors of $\mathrm{X}$ in both the midgut cell and the midgut lumen.

$\left(\right.$ Tyr $\left.^{4 \mathrm{~A}}-\mathrm{Glu}^{21 \mathrm{~A}}\right)$ of PCPBAe1 seems to be targeted primarily by the $\mathrm{E}$ protein, as shown by the significant deuterium exchange at this site, and further corroborated by the alanine site-directed mutagenesis of residues $\mathrm{Asp}^{18 \mathrm{~A}}$ and $\mathrm{Glu}{ }^{19 \mathrm{~A}}$. However, using the pro-region alone (ProCP) shows an extreme reduction in interaction ( 60 -fold reduction), suggesting that the conformational dynamics required for interaction of full-length PCPBAe1 might be different from the individual pro-region. The identified DENV E protein-interacting peptide regions of PCPBAe1 in other Ae. aegypti PCPS (PCPAe1 and PCPBAe111) are poorly conserved, suggesting that PCP homologs may possess different levels of inhibitory activities, if any, against DENV.

Furthermore, the HDXMS results suggest that PCPBAe1 binds across the E protein's dimeric interface by targeting flexible loop regions but not the conserved fusion loop (Asp ${ }^{98}$-Lys ${ }^{110}$ ). The $4 \mathrm{G} 2$ anti-DENV E protein antibody used in our ELISA experiment targets the conserved fusion loop epitope, consistent with the fact that the antibody detects DENV E-protein/virion/VLP even in the presence of PCPBAe1. In the previous docking studies by Tham et al, the authors predicted some of the residues of the $E$ protein $\left(\mathrm{Thr}^{66}\right.$, $\mathrm{Asn}^{67}, \mathrm{Val}^{251}$, and Lys $\left.{ }^{122}\right)$ that might interact with PCPBAe1 $(30,31)$. Our HDXMS analysis mapped all of these residues except Lys $^{122}$ (30, 31), and additional E protein amino acid stretches from domains I/II $\left(\right.$ Lys $^{64}-\mathrm{Glu}^{84}, \mathrm{Val}^{238}-\mathrm{Val}^{252}$, and Leu ${ }^{278}-$ Leu $^{287}$ ). Domain III, however, did not appear to contribute to the interaction with PCPBAe1. Thus, the individual domains alone are insufficient for interaction, as the full-length $\mathrm{E}$ protein shows a more substantial interaction. Targeting the individual E protein subunits or the mature/immature virion particle could lead to: (1) unavailability of E protein required for encapsulating newly formed viral nucleocapsids in the midgut, (2) prevent the E protein rearrangement and impede the maturation of viral particle or restrict the overall budding/exiting of viruses from the midgut cells, or (3) impede cellular binding/ endocytosis by binding of the mature CPBAe1 to the virus in the midgut lumen.

The interaction between PCPBAe1 and all DENV serotypes suggests that PCPBAe1 could potentially suppress all serotypes in the mosquito. There are three discontinuous interaction sites on the DENV E protein that bind to PCPBAe1. We systematically compared the sequences of these sites to understand the differential interactions (DENV-3 > DENV-2 > DENV-4 > DENV-1) with DENV serotypes. At first, we compared the sites of DENV-3 and DENV-1, which are at the opposite ends of affinities. Site-3 is completely conserved between DENV-3 and DENV-1, whereas site-2 shows a single residue change ( Thr $^{242}$ Asn) (Fig 7B). In contrast, site-1 shows several key differences. Specifically, the two hydrophilic Thr residues to hydrophobic residues in DENV-3 ( $T h r^{68}$ Ile and $T h r^{81} \mathrm{Val}$ ). These hydrophobic residues may enhance the interaction due to the 
tendency to bury themselves in the interaction surface. In addition, a Pro ${ }^{83}$ residue replaces $\mathrm{Val}^{83}$ in DENV-3, creating a proline bracket with $\mathrm{Pro}^{78}$ residue (32). Such proline brackets are known to enhance protein-protein interactions (33). Thus, it appears that the Site1 (62-82 residues) mostly defines the affinity differences observed between DENV-3 and DENV-1. There are several amino acid residue differences observed in all three sites between DENV-2 and DENV-4. Interestingly, sites-1 of both DENV-2 and DENV-4 have slightly shorter regions within the proline brackets than DENV-3. They also have a conserved Pro ${ }^{243}$ residue unlike DENV-1 and DENV-3. It is unclear which of these changes are responsible for the observed differences in affinity in these two serotypes.

Only the glycan motif at Asn ${ }^{67}$ on the E protein seems to influence interaction. The different glycan compositions at $\mathrm{Asn}^{67}$ between the insect and mammalian cell-purified viral antigens might partially account for the marginal differences observed in the interaction between PCPBAe1 and DENV (29). However, because this difference is only marginal (and not significant), these findings cannot wholly explain why PCPBAe1 only suppresses the release of DENV-2 in mosquito C6/36 cell line but not in a mammalian Vero cell line (7). Thus, apart from PCPBAe1 interaction with the E protein, other conditions in the mosquito cell line microenvironment such as cellular proteins/ receptors or attachment factors (34), that are different from the mammalian cell line microenvironment likely play a role in virus inhibition (Fig 8). For example, virus internalization might occur through distinct entry pathways, including clathrin-mediated or non-classical clathrin-independent endocytosis, depending on whether mosquito or human cell or specific host cells (34). Furthermore, C6/36 is a better cell line for DENV infection than Vero cell line (34).

In summary, we show that the residues $\mathrm{Asp}^{251}$ and $\mathrm{Ser}^{239}$ control substrate-specificity, and such residues may be targeted for developing anti-CPB molecules to arrest digestion and control mosquito reproduction. Because of its ability to bind all DENV serotypes, PCPBAe1 may also inhibit other DENV serotypes by targeting the E protein/virion, which could hamper viral packaging or morphogenesis, leading to an overall reduced viral maturation and release from the mosquito midgut. Alternatively, activated CPBAe1 may also inhibit mature DENV when released into DENV-infected blood in the midgut lumen, although this inhibition may be less than that of the full-length PCPBAe1. Besides, because the mature region usually degrades the pro-region of procarboxypeptidases upon tryptic cleavage, the independent proregion may not be available in the midgut when cleaved from the proenzyme (35). However, PCPBAe1 might be specific to DENV as we did not observe PCPBAe1 binding to CHIKV or MAYV VLPS.

Thus, our structural and functional studies of PCPBAe1 provide a blueprint for developing two distinct strategies: (i) carboxypeptidase inhibitors or antibodies as a strategy for mosquito population control and (ii) how PCPBAe1 elicits antiviral properties against DENV. However, it would be essential to evaluate the strategies aimed at blocking carboxypeptidase activities in DENV endemic areas to avoid compromising CPB antiviral activities in the mosquito. Moreover, even though PCPBAe1 serves as an anti-dengue viral molecule in the mosquito, it may not represent an effective dengue or other mosquito-borne viral diseases control strategy as the inhibition of viral propagation seems to only occur in the vector and may be specific to DENV.

\section{Materials and Methods}

\section{Cloning, recombinant protein expression and purification}

\section{PCPBAe1 and E protein domain I/II and III expression and purification from the $\mathrm{E}$. coli system}

The gene coding for proenzyme Aedes aegypti procarboxypeptidase B1 (PCPBAe1) (Genbank accession number: Q6J661) was codon optimized and synthesized by Genscript for recombinant protein expression in E. coli (BL-21(DE3)) (shuffle) cells. The gene was subcloned into pGEX-6p-1 vector (Novagen) using the Bamh1/Xho1 restriction sites to form the pGEX-precision protease site-PCPBAe1 fusion construct. Confirmed fusion plasmid was transformed into the E. coli shuffle (BL-21(DE3)) cells. Transformed cells were inoculated into $10 \mathrm{ml}$ LB medium supplemented with $100 \mu \mathrm{g} / \mathrm{ml}$ ampicillin and grown at $37^{\circ} \mathrm{C}$ overnight as a primary culture inoculum. The overnight culture was used to inoculate $1 \mathrm{~L}$ flask secondary LB medium and grown until the optical density (OD) reached 0.8 . The culture was cooled at room temperature and induced with $0.3 \mathrm{mM} \mathrm{IPTG}$, supplemented with $60 \mu \mathrm{M}$ zinc sulphate. The induced culture was shaken at $18^{\circ} \mathrm{C}, 150 \mathrm{rpm}$ overnight. Overnight bacteria culture was pelleted down at 4,000 rpm (centrifuge rotor ID: JLA-8.1000) for $30 \mathrm{~min}$. Bacteria cell pellets were resuspended in $35 \mathrm{ml} / \mathrm{l}$ pellet in lysis buffer containing $50 \mathrm{mM}$ citrate buffer, pH 6.0, $0.3 \mathrm{M} \mathrm{NaCl}, 5 \%$ glycerol $1 \mathrm{mM}$ DT, and a protease cocktail inhibitor tablet (Roche). Pellets were vortexed and sonicated for three cycles ( 5 min, $1 \mathrm{~s}$ on, $2 \mathrm{~s}$ off). Sample was then span down at 18,000 rpm (centrifuge rotor ID: JA-20) for $30 \mathrm{~min}$ at $4^{\circ} \mathrm{C}$ and bound to GST beads (GE) previously equilibrated with the same lysis buffer at $4^{\circ} \mathrm{C}$ for $4 \mathrm{~h}$ for affinity purification. Beads were washed three times with $90 \mathrm{ml}$ of lysis buffer each time and incubated overnight with elution buffer supplemented with PreScission Protease (GE) to cleave the GST tag. First step purification of anion exchange chromatography was done on QHP column (GE), applying a gradient from 100\% buffer A (20 Mm Tris, pH 8.0, $1 \mathrm{mM}$ DTT) to $100 \%$ buffer B (20 mM Tris/ $\mathrm{NaCl} 1 \mathrm{M}, \mathrm{pH} 8.0,1 \mathrm{mM}$ DTT) in $30 \mathrm{~min}$. A final size exclusion chromatography on a Superdex S-200 column (GE) (20 mM sodium citrate pH 6.0, $0.1 \mathrm{M} \mathrm{NaCl}$, 5\% glycerol, and 1 mM DTT) was performed to achieve maximal purity. The peak fractions were collected and concentrated to $10 \mathrm{mg} / \mathrm{ml}$. All the other PCPBAe1 mutants were purified using the E. coli expression and purified as above.

DENV-2 E protein domains I/II (a.a 1-296) and domain III (a.a 296-394) were cloned separately into a modified pet23b vector carrying sumo-tag and modified pet32a vector carrying only $6 \mathrm{X}-$ Histidine tag. Protein expression conditions were similar to the above described. Purification was carried out with nickel beads affinity chromatography, followed by ion exchange chromatography (buffer similar to above described) and gel filtration in the buffer condition (Tris, pH 8.0, $150 \mathrm{mM} \mathrm{NaCl}, 5 \%$ glycerol, and $1 \mathrm{Mm}$ DTT). The tags were not cleaved before further assays.

\section{PCPBAe1 expression and purification from the insect baculoviral system}

The PCPBAe1 construct was further cloned into pFastBac1 vector with $6 \mathrm{X}$-Histidine purification tag for protein expression in insectcell baculovirus expression system using Spodoptera frugiperda 
(SF9) cells by following previous protocols $(36,37)$. Protein was secreted into the culture medium and purified using nickel-NTA beads and gel filtration chromatography. Dynamic light scattering studies were carried out on a DynaPro Light Scattering instrument (Protein Solutions) to assess protein quality.

\section{Enzymatic assay of CPBAe1}

The proenzyme PCPBAe1 was activated using trypsin limited proteolysis into the active form CPBAe1 and further purified using gel filtration and ion exchange chromatography. CPBAe1 activity was determined by the continuous spectrophotometric rate determination method of Folk et al (1960) where the reaction velocity is determined by an increase in absorbance at $254 \mathrm{~nm}$ resulting from the hydrolysis of Hippuryl-L-Arginine (Hip-L-Arg), Hip-L-Lys, and Hip-L-Phe. One unit causes the hydrolysis of $1 \mu \mathrm{mol}$ of Hippuryl-Larginine per minute at $25^{\circ} \mathrm{C}$ and $\mathrm{pH} 7.65$ under the specified conditions. Briefly, substrates at different concentrations (0.05-10 mM) were diluted in buffer $(0.025 \mathrm{M}$ Tris- $\mathrm{HCl}, \mathrm{pH} 7.65$, containing $0.1 \mathrm{M}$ sodium chloride). Different concentrations of stock trypsin activated CPBAe1 solution were diluted in assay buffer (0.025 M Tris- $\mathrm{HCl}$ buffer, $\mathrm{pH} 7.65$, containing $0.1 \mathrm{M} \mathrm{NaCl}$ ). The spectrophotometer was set at $254 \mathrm{~nm}$ and $25^{\circ} \mathrm{C}$. Next, $2.9 \mathrm{ml}$ of substrate solution was pipetted into two separate quartz cuvettes and incubated in spectrophotometer at $25^{\circ} \mathrm{C}$ for $3-4 \mathrm{~min}$ to reach temperature equilibration and blank rate was established. To start activity measurement, $0.1 \mathrm{ml}$ of diluted enzyme was added to the substrate in the cuvette and the increase in absorbance (A254) was recorded for 3-4 $\mathrm{min}$. The fastest linear rate $(\triangle \mathrm{A} 254 \mathrm{~nm} /$ minute) over a 1-min interval for the test and the blank reactions was then determined from the initial linear portion of the curve and used to calculate the specific enzyme activity values. All the OD readings were done in triplicates. The $\mathrm{Km}$ was also calculated using a kinetics plot.

\section{Crystallization and structure determination of proenzyme PCPBAe1}

Crystallization screens were conducted using the sitting drop vapor diffusion method at room temperature $\left(22^{\circ} \mathrm{C}\right)$ by mixing protein with the crystallization reservoir solutions at a 1:1 volume ratio. Crystals appeared after about 2 wk in the condition Crystal Screen $1(0.2 \mathrm{M}$ ammonium sulphate, $0.1 \mathrm{M}$ sodium cacodylate trihydrate, $\mathrm{pH}$ 6.5, 30\% wt/vol PEG 8000) (Hampton Research). The diffraction quality crystals were immersed in mother liquor solution supplemented with $25 \%$ ethylene glycol as a cryoprotectant for data collection. A complete native $2.0 \AA$ resolution diffraction data set was collected at $100 \mathrm{~K}$ using Rigaku MicroMax-007 HF equipped with Saturn 944+ CCD detector, National University of Singapore. Data were processed with the HKL-2000 program (38). The structure was determined using molecular replacement (39) using the coordinates of CPAHa (PDB: 1JQG) (10) and the final model was refined at $2.08 \AA$ resolution. The structure has good stereochemical parameters evaluated with PROCHECK (Table S2). PyMOL and CHIMERA (40) were used to prepare all structure-related figures.
ELISA assay to characterize PCPBAe1-DENV virion, E protein and VLP interaction

High binding 96-well plates (Thermo Fisher Scientific) were coated overnight at $4^{\circ} \mathrm{C}$ with a $5 \mu \mathrm{g}$ of all PCPBAe 1 constructs and mutants or $5 \mu \mathrm{g}$ BSA or sumo tag protein alone in coating buffer. All samples were coated in triplicates. The next day, plates were washed twice with PBS and blocked with blocking buffer (1\% BSA in 1XPBST) for $1 \mathrm{~h}$ at $28^{\circ} \mathrm{C}$. Plates were incubated with $100 \mu \mathrm{l}$ of DENV-2 VLPS/ infectious virion serotypes (DENV-1, DENV-2, DENV-3, and DENV-4) $\left(2 \times 10^{5}\right.$ p.f.u $)$ or recombinant DENV-2 E protein $(1 \mu \mathrm{g})$ (full-length, sumo-tagged domain $\mathrm{I} / \mathrm{II}$, or domain III) at $28^{\circ} \mathrm{C}$ for $1 \mathrm{~h}$, considering the temperature of the mosquito vector. DENV-2 E protein (DENV-2ENV-100) and VLPS (DENV-2-VLP-100, CHIKV-VLP-100, and MAYV-VLP: REC31616-100) were purchased from The Native Antigens Company (purified from HEK293 cell cultures), whereas infectious virion was obtained from our collaborators (A/P Sylvie Alonso, National University of Singapore). Unbound proteins/virion were washed three times with buffer ( $1 \times$ PBST $+0.01 \%$ Tween 20) and incubated with $100 \mu \mathrm{l}$ of $1 \mu \mathrm{g} / \mathrm{ml}$ mouse anti-DENV-2 primary antibody (MAB10216, Millipore or anti-E protein domain III [2D73], absolute antibody) for $2 \mathrm{~h}$ at RT to detect VLP/E protein/virion. Unbound primary antibody was then washed three times with buffer (1x PBST $+0.01 \%$ Tween 20) and plates incubated with $100 \mu \mathrm{l}$ of 1:5,000 dilution rabbit anti-mouse HRP conjugated secondary antibody and washed six times after $2 \mathrm{~h}$ of incubation at RT. Reaction was visualized after incubating with $80 \mu \mathrm{l}$ of tetramethylbenzidine (TMB). After $5 \mathrm{~min}$, the reaction was stopped with $1 \mathrm{M} \mathrm{HCl}$ and plates were read at $450 \mathrm{~nm}$ in Tecan Infinite $\mathbf{2 0 0}$ pro (Life Sciences and Diagnostics).

\section{Hydrogen-deuterium exchange (HDXMS) of PCPBAe1-E protein interaction}

Interaction of PCPBAe1 and DENV-2 E protein were mapped by amide hydrogen-deuterium exchange mass spectrometry (HDXMS) (41). HDX reaction was initiated by diluting the protein in $90 \mu \mathrm{l}$ deuterated buffer (50 mM PBS pH 7.4) to a final 90\% $\mathrm{D}_{2} \mathrm{O}$ (Cambridge Isotopes) concentration. For HDXMS of free proteins, $3 \mu \mathrm{l}$ of $1 \mathrm{mg} / \mathrm{ml}$ of DENV-2 E protein and $5 \mu \mathrm{l}$ of $1 \mathrm{mg} / \mathrm{ml}$ of PCPBAe 1 were used. For HDXMS of complex, the proteins were mixed in 1:2 $\mathrm{E}$ protein: PCPBAe1 stoichiometric ratio and incubated for $20 \mathrm{~min}$ before deuterium labeling reaction. Deuterium labeling was carried out for 1 - and 10 -min time points at $28^{\circ} \mathrm{C}$ temperature, similar to mosquito vector. The exchange reaction was stopped by lowering the $\mathrm{pH}$ to $\sim 2.6$ by addition of $100 \mu \mathrm{l}$ quench solution (0.8 M guanidinium hydrochloride, $0.25 \mathrm{M} \mathrm{TCEP}$ ) and temperature to $0^{\circ} \mathrm{C}$ to minimize back-exchange. Non-deuterated control experiments of PCPBAe1 and $E$ protein alone were also carried out by diluting the samples in aqueous buffer, followed by quench solution.

The quenched samples were incubated on ice $\left(0^{\circ} \mathrm{C}\right)$ for $30 \mathrm{~s}$ followed by 3 min proteolytic digestion by immobilized pepsin and the digested pepsin-cleaved fragments ("peptides") were resolved by reverse-phase chromatography using C18 column. The peptides were eluted using a $10 \mathrm{~min} 8-40 \%$ gradient of $0.1 \%$ formic acid in acetonitrile, pumped at $40 \mu \mathrm{l} / \mathrm{min}$ by nanoACQUITY binary solvent manager (42). The peptides eluted were then subjected to mass 
analysis by injecting onto a coupled high resolution Synapt G2-Si mass spectrometer and identified in HDMSe mode, as described previously (41).

Mass spectra of non-deuterated controls were used for peak identification and peptide matching using ProteinLynx Global Server v3.0.1 software (Waters) (43) against individual databases consisting amino acid sequences of PCPBAe1 and DENV-2 E protein. Peptides were filtered and only high signal-to-noise ratio nonoverlapping peptides were selected for analysis of deuteration by DynamX v3.0 (Waters). All values reported are an average of three deuterium exchange reactions and are not corrected for back-exchange. The absolute difference in deuterons exchanged by all peptides relative to another condition is represented as a difference plot listed from the $\mathrm{N}$ - to $\mathrm{C}$ terminus.

\section{Site-directed mutagenesis}

For the activity assays, three mutants were generated: $\mathrm{Asp}^{251} \mathrm{Glu}$, $\mathrm{Ser}^{239} \mathrm{Gly}$, and $\mathrm{Asp}^{251} \mathrm{Gly}+\mathrm{Ser}^{239} \mathrm{Gly}$. Based on our HDXMS results, four PCPBAe1 mutants were generated including Asp ${ }^{18 \mathrm{~A}}$ and $\mathrm{Glu}^{19 \mathrm{~A}}$ deletion, linker region (Glu $\left.{ }^{85} \mathrm{Ala} \mathrm{Arg}^{87} \mathrm{AlaArg}{ }^{89} \mathrm{Ala}\right)$ and $\left(\mathrm{Glu}^{168} \mathrm{Ala}\right.$, Glu ${ }^{170} \mathrm{Ala}$, and $\left.\mathrm{Arg}^{172} \mathrm{Ala}\right)$ alanine mutagenesis, and $\mathrm{Val}^{300}-\mathrm{Phe}^{305}$ deletion. All the primer/oligonucleotide information are provided in Table S3. All the PCR reactions were carried out using KAPABIOSYSTEMS HiFi PCR reaction Kits (Roche).

\section{Generation of de-glycosylated DENV-2 mutant}

D2Y98P-PP1 (GenBank: JF327392.1) RNA genome was extracted using QIAamp Viral RNA Kits (QIAGEN). cDNA synthesis was performed using GoScript Reverse Transcriptase (Promega) as per manufacturer's instructions. Four PCR fragments of around 2,700 nucleotides long were generated from CDNA using primer pairs with NEB Q5 Hot-Start highfidelity 2x Master Mix (New England Biolabs). Fragments were gelpurified with MinElute gel extraction Kit (QIAGEN) after agarose gel electrophoresis and blunt end cloning was performed into pCR-Blunt IITOPO Vector (Invitrogen). Site-directed mutagenesis was performed on plasmid that carried E gene to introduce the desired mutation using primer pairs. The mutated gene was amplified with other genes fragments via PCR and seamlessly assembled with vector contains CMV promoter sequence, hepatitis delta virus ribozyme, and simian virus 40 (SV40) poly-A sequence using NEBuilder HiFi DNA Assembly Master Mix (New England Biolabs) at $50^{\circ} \mathrm{C}$ for 60 min. This CMV-DENV genome-HDVr-SV40pA assembled product was transfected into BHK-21 cells using Lipofectamine 2000 (Invitrogen). Four to 6 d later, the viral supernatant was collected and sequenced for validation using Sanger sequencing. All the primer/oligonucleotide information are provided in Table \$4.

\section{Data Deposition}

Three dimensional atomic coordinates, and structure factors of PCPBAe1 have been deposited in the Protein Data Bank, www.pdb.org (PDB ID codes 7EQX).

\section{Supplementary Information}

Supplementary Information is available at https://doi.org/10.26508/lsa 202101211.

\section{Acknowledgements}

This work was supported by Ministry of Education, Singapore (MoE Tier-3) grant (R154-000-697-112) and R154-000-C07-114 (ACRF Tier 1 grant), respectively. E Gavor is a graduate scholar in receipt of the Singapore International Graduate Award (SINGA) research scholarship.

\section{Author Contributions}

E Gavor: conceptualization, data curation, software, formal analysis, validation, visualization, methodology, and writing-original draft, review, and editing.

YK Choong: data curation, software, formal analysis, validation, visualization, methodology, and writing-review and editing.

NK Tulsian: data curation, software, formal analysis, validation, visualization, methodology, and writing-review and editing. D Nayak: software, formal analysis, validation, and visualization. F Idris: formal analysis, validation, investigation, methodology, and writing-review and editing.

H Sivaraman: methodology.

DHR Ting: methodology.

A Sylvie: resources, formal analysis, and writing-review and editing. YK Mok: writing-review and editing.

RM Kini: formal analysis, validation, visualization, and writing-review and editing.

J Sivaraman: conceptualization, resources, software, formal analysis, supervision, funding acquisition, validation, project administration, and writing-original draft, review, and editing.

\section{Conflict of Interest Statement}

The authors declare that they have no conflict of interest.

\section{References}

1. Franz AW, Kantor AM, Passarelli AL, Clem RJ (2015) Tissue barriers to arbovirus infection in mosquitoes. Viruses 7: 3741-3767. doi:10.3390/ v7072795

2. Kato N, Mueller CR, Fuchs JF, McElroy K, Wessely V, Higgs S, Christensen BM (2008) Evaluation of the function of a type I peritrophic matrix as a physical barrier for midgut epithelium Invasion by mosquito-borne pathogens in Aedes aegypti. Vector Borne Zoonotic Dis 8: 701-712. doi:10.1089/vbz.2007.0270

3. Edwards MJ, Lemos FJ, Donnelly-Doman M, Jacobs-Lorena M (1997) Rapid induction by a blood meal of a carboxypeptidase gene in the gut of the mosquito Anopheles gambiae. Insect Biochem Mol Biol 27: 1063-1072. doi:10.1016/S0965-1748(97)00093-3

4. Lavazec C, Boudin C, Lacroix R, Bonnet S, Diop A, Thiberge S, Boisson B, Tahar R, Bourgouin C (2007) Carboxypeptidases B of Anopheles gambiae as targets for a Plasmodium falciparum transmission-blocking vaccine. Infect Immun 75: 1635-1642. doi:10.1128/IAl.00864-06 
5. Raz A, Dinparast Djadid N, Zakeri S (2013) Molecular characterization of the carboxypeptidase B1 of anopheles stephensi and its evaluation as a target for transmission-blocking vaccines. Infect Immun 81: 2206-2216. doi:10.1128/IAl.01331-12

6. Tham HW, Balasubramaniam VR, Chew MF, Ahmad H, Hassan SS (2015) Protein-protein interactions between $A$. aegypti midgut and dengue virus 2: Two-hybrid screens using the midgut cDNA library. I Infect Dev Ctries 9: 1338-1349. doi:10.3855/jidc.6422

7. Tham HW, Balasubramaniam VR, Tejo BA, Ahmad H, Hassan SS (2014) CPB1 of Aedes aegypti interacts with DENV2 E protein and regulates intracellular viral accumulation and release from midgut cells. Viruses 6: 5028-5046. doi:10.3390/v6125028

8. Bown DP, Gatehouse JA (2004) Characterization of a digestive carboxypeptidase from the insect pest corn earworm (Helicoverpa armigera) with novel specificity towards C-terminal glutamate residues. Eur J Biochem 271: 2000-2011. doi:10.1111/j.1432-1033.2004.04113.x

9. Sui YP, Liu XB, Chai LQ, Wang JX, Zhao XF (2009) Characterization and influences of classical insect hormones on the expression profiles of a molting carboxypeptidase A from the cotton bollworm (Helicoverpa armigera). Insect Mol Biol 18: 353-363. doi:10.1111/j.13652583.2009.00879.x

10. Estébanez-Perpiñá E, Bayés A, Vendrell J, Jongsma MA, Bown DP, Gatehouse JA, Huber R, Bode W, Avilés FX, Reverter D (2001) Crystal structure of a novel mid-gut procarboxypeptidase from the cotton pest Helicoverpa armigera. J Mol Biol 313: 629-638. doi:10.1006/jmbi.2001.5076

11. Bayés A, Comellas-Bigler M, Rodríguez de la Vega M, Maskos K, Bode W, Aviles FX, Jongsma MA, Beekwilder J, Vendrell I (2005) Structural basis of the resistance of an insect carboxypeptidase to plant protease inhibitors. Proc Natl Acad Sci U S A 102: 16602-16607. doi:10.1073/ pnas. 0505489102

12. Sapio MR, Fricker LD (2014) Carboxypeptidases in disease: Insights from peptidomic studies. Proteomics Clin Appl 8: 327-337. doi:10.1002/ prca.201300090.Carboxypeptidases

13. Moskalyk LA (1998) Carboxypeptidase B in Anopheles gambiae (Diptera: Culicidae): Effects of abdominal distention and blood ingestion. J Med Entomol 35: 216-221. doi:10.1093/jmedent/35.3.216

14. Kumar A, Sharma A, Sharma R, Gakhar SK (2014) Identification, characterization and analysis of expression of gene encoding carboxypeptidase A in Anopheles culicifacies A (Diptera: Culicidae). Acta Trop 139: 123-130. doi:10.1016/j.actatropica.2014.05.022

15. Cui Y, Niu G, Li VL, Wang X, Li J (2020) Analysis of blood-induced Anopheles gambiae midgut proteins and sexual stage Plasmodium falciparum interaction reveals mosquito genes important for malaria transmission. Sci Rep 10: 14316-14412. doi:10.1038/s41598-020-71186-5

16. Adedeji EO, Ogunlana OO, Fatumo S, Beder T, Ajamma Y, Koenig R, Adebiyi E (2020) Anopheles metabolic proteins in malaria transmission, prevention and control: A review. Parasit Vectors, 13: 465-530. doi:10.1186/s13071-020-04342-5

17. Masuyer G, Akif M, Czarny B, Beau F, Schwager SL, Sturrock ED, Isaac RE, Dive V, Acharya KR (2014) Crystal structures of highly specific phosphinic tripeptide enantiomers in complex with the angiotensin-I converting enzyme. FEBS / 281: 943-956. doi:10.1111/febs.12660

18. Mairiang D, Zhang H, Sodja A, Murali T, Suriyaphol P, Malasit P, Limjindaporn T, Finley RL (2013) Identification of new protein interactions between dengue fever virus and its hosts, human and mosquito. PLoS One 8: e53535. doi:10.1371/journal.pone.0053535

19. Moreira LA, Edwards MJ, Adhami F, Jasinskiene N, James AA, JacobsLorena M (2000) Robust gut-specific gene expression in transgenic Aedes aegypti mosquitoes. Proc Natl Acad Sci U S A 97: 10895-10898. doi:10.1073/pnas.97.20.10895

20. Coll M, Guasch A, Avilés FX, Huber R (1991) Three-dimensional structure of porcine procarboxypeptidase B: A structural basis of its inactivity. EMBO / 10: 1-9. doi:10.1002/j.1460-2075.1991.tb 07914.x
21. Garcia-Guerrero MC, Garcia-Pardo J, Berenguer E, Fernandez-Alvarez R, Barfi GB, Lyons PJ, Aviles FX, Huber R, Lorenzo J, Reverter D (2018) Crystal structure and mechanism of human carboxypeptidase O: Insights into its specific activity for acidic residues. Proc Natl Acad Sci U S A 115: E3932-E3939. doi:10.1073/pnas.1803685115

22. Vendrell J, Querol E, Avilés FX (2000) Metallocarboxypeptidases and their protein inhibitors. Structure, function and biomedical properties. Biochim Biophys Acta 1477: 284-298. doi:10.1016/S0167-4838(99)00280-0

23. Tan AK, Eaton DL (1995) Activation and characterization of procarboxypeptidase B from human plasma. Biochemistry 34: 5811-5816. doi:10.1021/bi00017a012

24. Arolas JL, Lorenzo J, Rovira A, Vendrell J, Aviles FX, Ventura S (2004) Secondary binding site of the potato carboxypeptidase inhibitor Contribution to its structure, folding, and biological properties. Biochemistry 43: 7973-7982. doi:10.1021/bi049596j

25. Valnickova Z, Sanglas L, Arolas JL, Petersen SV, Schar C, Otzen D, Aviles FX, Gomis-Rüth FX, Enghild JJ (2010) Flexibility of the thrombinactivatable fibrinolysis inhibitor pro-domain enables productive binding of protein substrates. J Biol Chem 285: 38243-38250. doi:10.1074/jbc.M110.150342

26. Titani K, Ericsson LH, Walsh KA, Neurath H (1975) Amino-acid sequence of bovine carboxypeptidase B. Proc Natl Acad Sci U S A 72: 1666-1670. doi:10.1073/pnas.72.5.1666

27. Aloy P, Companys V, Vendrell J, Aviles FX, Fricker LD, Coll M, Gomis-Rüth FX (2001) The crystal structure of the inhibitor-complexed carboxypeptidase $\mathrm{D}$ domain II and the modeling of regulatory carboxypeptidases. J Biol Chem 276: 16177-16184. doi:10.1074/ jbc.M011457200

28. Pokidysheva E, Zhang Y, Battisti AJ, Bator-Kelly CM, Chipman PR, Xiao C, Gregorio GG, Hendrickson WA, Kuhn RJ, Rossmann MG (2006) Cryo-EM reconstruction of dengue virus in complex with the carbohydrate recognition domain of DC-SIGN. Cell 124: 485-493. doi:10.1016/ j.cell.2005.11.042

29. Hacker K, White L, de Silva AM (2009) N-linked glycans on dengue viruses grown in mammalian and insect cells. J Gen Virol 90: 2097-2106. doi:10.1099/vir.0.012120-0

30. Christian EA, Kahle KM, Mattia K, Puffer BA, Pfaff JM, Miller A, Paes C, Davidson E, Doranz BJ (2013) Atomic-level functional model of dengue virus envelope protein infectivity. Proc Natl Acad Sci U S A 110: 18662-18667. doi:10.1073/pnas.1310962110

31. Modis Y, Ogata S, Clements D, Harrison SC (2003) A ligand-binding pocket in the dengue virus envelope glycoprotein. Proc Natl Acad Sci U S A 100: 6986-6991. doi:10.1073/pnas.0832193100

32. Kini RM, Evans HJ (1995) A hypothetical structural role for proline residues in the flanking segments of protein-protein interaction sites. Biochem Biophys Res Commun 212: 1115-1124. doi:10.1006/ bbrc.1995.2084

33. Kini RM, Evans HJ (1995) A novel approach to the design of potent bioactive peptides by incorporation of proline brackets: Antiplatelet effects of arg-gly-asp peptides. FEBS Lett 375: 15-17. doi:10.1016/00145793(95)01144-4

34. Reyes-del Valle J, Salas-Benito J, Soto-Acosta R, del Angel RM (2014) Dengue virus cellular receptors and tropism. Curr Trop Med Rep 1:36-43. doi:10.1007/s40475-013-0002-7

35. Villegas V, Vendrell J, Avilés X (1995) The activation pathway of procarboxypeptidase $B$ from porcine pancreas: Participation of the active enzyme in the proteolytic processing. Protein Sci 4: 1792-1800. doi:10.1002/pro.5560040914

36. Usami A, Ishiyama S, Enomoto C, Okazaki H, Higuchi K, Ikeda M, Yamamoto T, Sugai M, Ishikawa Y, Hosaka Y, et al (2011) Comparison of recombinant protein expression in a baculovirus system in insect cells (Sf9) and silkworm. J Biochem 149: 219-227. doi:10.1093/ $\mathrm{jb} / \mathrm{mvq138}$ 
37. Scholz J, Suppmann S (2017) A new single-step protocol for rapid baculovirus-driven protein production in insect cells. BMC Biotechnol 17: 83-89. doi:10.1186/s12896-017-0400-3

38. Powell HR (2017) X-ray data processing. Biosci Rep 37: 1-14. doi:10.1042/ BSR20170227

39. McCoy AJ (2006) Solving structures of protein complexes by molecular replacement with Phaser. Acta Crystallogr D Biol Crystallogr 63: 32-41. doi:10.1107/S0907444906045975

40. Mooers BH (2016) Simplifying and enhancing the use of PyMOL with horizontal scripts. Protein Sci 25: 1873-1882. doi:10.1002/pro.2996

41. Raghuvamsi PV, Tulsian NK, Samsudin F, Qian X, Purushotorman K, Yue G, Kozma MM, Hwa WY, Lescar J, Bond PJ, et al (2021) Sars-cov-2 s protein: Ace2 interaction reveals novel allosteric targets. Elife 10: 1-47. doi:10.7554/eLife.63646
42. Wales1 TE, Fadgen KE, Gerhardt GC, Engen JR (2011) High-speed and highresolution UPLC separation at zero degrees celsius. Bone 23: 1-7. doi:10.1021/ac8008862.High-speed

43. Li GZ, Vissers JP, Silva JC, Golick D, Gorenstein MV, Geromanos SJ (2009) Database searching and accounting of multiplexed precursor and product ion spectra from the data independent analysis of simple and complex peptide mixtures. Proteomics 9: 1696-1719. doi:10.1002/ pmic.200800564

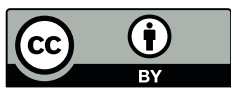

License: This article is available under a Creative Commons License (Attribution 4.0 International, as described at https://creativecommons.org/ licenses/by/4.0/). 